\title{
Rosenthal operator spaces
}

\author{
by \\ M. Junge (Urbana, IL), N. J. Nielsen (Odense) \\ and T. Oikhberg (Irvine, CA)
}

\begin{abstract}
In 1969 Lindenstrauss and Rosenthal showed that if a Banach space is isomorphic to a complemented subspace of an $L_{p}$-space, then it is either an $\mathcal{L}_{p}$-space or isomorphic to a Hilbert space. This is the motivation of this paper where we study nonHilbertian complemented operator subspaces of non-commutative $L_{p}$-spaces and show that this class is much richer than in the commutative case. We investigate the local properties of some new classes of operator spaces for every $2<p<\infty$ which can be considered as operator space analogues of the Rosenthal sequence spaces from Banach space theory, constructed in 1970. Under the usual conditions on the defining sequence $\sigma$ we prove that most of these spaces are operator $\mathcal{L}_{p}$-spaces, not completely isomorphic to previously known such spaces. However, it turns out that some column and row versions of our spaces are not operator $\mathcal{L}_{p}$-spaces and have a rather complicated local structure which implies that the Lindenstrauss-Rosenthal alternative does not carry over to the non-commutative case.
\end{abstract}

Introduction. In 1970 Rosenthal [26] constructed new examples of $\mathcal{L}_{p^{-}}$ spaces for every $2 \leq p<\infty$ using probabilistic methods now famous as the Rosenthal inequalities. These methods were later used by Bourgain, Rosenthal and Schechtman [3] to construct an uncountable family of mutually non-isomorphic $\mathcal{L}_{p^{-}}$-spaces.

In the framework of operator spaces a theory of operator $\mathcal{L}_{p^{-}}$-spaces, called $\mathcal{O} \mathcal{L}_{p^{-}}$-spaces, is now being developed (see e.g. [4] and [11]). These are spaces where the operator space structure of the finite-dimensional subspaces is determined by a system of finite-dimensional non-commutative $L_{p}$-spaces. If in a given space these $L_{p}$-spaces can be chosen to be completely complemented, the space is called a $\mathcal{C O} \mathcal{L}_{p}$-space. If they can be chosen to be $S_{p}^{n}$ 's $\left(S_{p}\right.$ denotes the Schatten $p$-class), then the space is called an $\mathcal{O S}_{p}$-space,

2000 Mathematics Subject Classification: 46B20, 46L07, 46L52.

Key words and phrases: non-commutative $L_{p}$-spaces, $\mathcal{O} \mathcal{L}_{p}$-spaces.

Research of M. Junge supported by NSF grant DMS-0301116 and DMS 05-56120.

Research of N. J. Nielsen supported by the Danish Natural Science Research Council, grant 21020436.

Research of T. Oikhberg supported by NSF grant DMS-0500957. 
and a $\mathcal{C O S} \mathcal{S}_{p}$-space if the $S_{p}^{n}$ 's can be chosen completely complemented. In the present paper we consider some operator space analogues of the Rosenthal spaces, sequence spaces as well as matricial analogues.

For a given $2<p<\infty$ and a given strictly positive sequence $\sigma=\left(\sigma_{n}\right)$ we construct three families of operator spaces, a sequence space family consisting of spaces called $X_{p}(\sigma), X_{p, r_{p}}(\sigma)$, and $X_{p, c_{p}}(\sigma)$, and two families of matricial operator spaces. All the spaces are mutually non-completely isomorphic as operator spaces, but the spaces in each family are isomorphic to each other as Banach spaces; the three sequence spaces are actually Banach space isomorphic to the original Rosenthal sequence space. One of our main results states that if $2<p<\infty, \sigma_{n} \rightarrow 0$, and $\sum_{n=1}^{\infty} \sigma_{n}^{2 p /(2 p-2)}=\infty$, then $X_{p, c_{p}}(\sigma)$ is completely complemented in a non-commutative $L_{p}$-space and contains $\ell_{p}$ cb-complemented. However $X_{p, c_{p}}(\sigma)$ is not an $\mathcal{O} \mathcal{L}_{p}$-space. Similarly for $X_{p, r_{p}}(\sigma)$. This shows that the Lindenstrauss-Rosenthal alternative [17] does not carry over to the non-commutative case.

We now wish to discuss the arrangement of this paper in greater detail. In Section 1 we construct our spaces, investigate their basic properties and prove among other things that under the above conditions on $\sigma$ the three sequence spaces are unique up to complete isomorphisms (in analogy with Rosenthal's result). In Section 2 we make a detailed investigation of the local structure of the spaces $X_{p}(\sigma), X_{p, c_{p}}(\sigma)$, and $X_{p, r_{p}}(\sigma)$ and prove that $X_{p}(\sigma)$ is an $\mathcal{O} \mathcal{L}_{p}$-space while $X_{p, r_{p}}(\sigma)$ and $X_{p, c_{p}}(\sigma)$ are not. We also show that some combinations of the different spaces cannot be paved with local pieces of each other. This implies that a general structure theory for completely complemented non-Hilbertian subspaces of non-commutative $L_{p}$-spaces is out of reach for the moment (see e.g. Proposition 2.19 and Remark 2.20). Section 3 is devoted to the study of the matricial spaces; we show that they are all $\mathcal{O S}_{p^{-}}$-spaces and that $Y_{p}(\sigma)$ is cb-complemented in $L_{p}(\mathcal{R})(\mathcal{R}$ the hyperfinite type $\mathrm{II}_{1}$ factor) while $Z_{p}(\sigma)$ does not cb-embed into $L_{p}(\mathcal{R})$. In Section 4 we prove that certain $\mathcal{O} \mathcal{L}_{p}$-spaces contain cb-uncomplemented copies of themselves.

0. Notation and preliminaries. In this paper we shall use the notation and terminology commonly used in the theory of operator algebras, operator spaces and Banach space theory as it appears in [5], [11], [18], [19], [23] and [28].

If $H$ is a Hilbert space, we let $B(H)$ denote the space of all bounded operators on $H$ and for every $n \in \mathbb{N}$ we let $M_{n}$ denote the space of all $n \times n$ matrices of complex numbers, i.e. $M_{n}=B\left(\ell_{2}^{n}\right)$. If $X$ is a subspace of some $B(H)$ and $n \in \mathbb{N}$, then $M_{n}(X)$ denotes the space of all $n \times n$ matrices with $X$-valued entries which we in the natural manner consider as a subspace of 
$B\left(\ell_{2}^{n}(X)\right)$. An operator space $X$ is a norm closed subspace of some $B(H)$ equipped with the distinguised matrix norm inherited by the spaces $M_{n}(X)$, $n \in \mathbb{N}$. An abstract matrix norm characterization of operator spaces was given by Ruan (see e.g. [5]).

If $X$ and $Y$ are operator spaces, then a linear operator $T: X \rightarrow Y$ is called completely bounded (for short, cb-bounded) if the corresponding linear maps $T_{n}: M_{n}(X) \rightarrow M_{n}(Y)$ are uniformly bounded in $n$, i.e.

$$
\|T\|_{\mathrm{cb}}=\sup \left\|T_{n}\right\|<\infty .
$$

The space of all completely bounded operators from $X$ to $Y$ will be denoted by $C B(X, Y)$.

It follows from [5] that a linear functional on an operator space $X$ is bounded if and only if it is cb-bounded, and its cb-norm and operator norm coincide. This defines an operator structure on $X^{*}$ so that isometrically we have $M_{n}\left(X^{*}\right)=C B\left(X, M_{n}\right)$ for all $n \in \mathbb{N}$.

An operator is a complete contraction, respectively a complete isometry, or a complete quotient if $\|T\|_{\mathrm{cb}} \leq 1$, respectively if each $T_{n}$ is an isometry, or a quotient map. An operator $T$ is called a complete isomorphism (for short, a cb-isomorphism) if it is a completely bounded linear isomorphism with a completely bounded linear inverse. If $X$ and $Y$ are cb-isomorphic operator spaces we put

$$
d_{\mathrm{cb}}(X, Y)=\inf \left\{\|T\|_{\mathrm{cb}}\left\|T^{-1}\right\|_{\mathrm{cb}} \mid T \text { is a cb-isomorphism from } X \text { to } Y\right\},
$$

which is called the completely bounded Banach-Mazur distance (for short, the $c b$-distance) between $X$ and $Y$.

We let $S_{\infty} \subseteq B\left(\ell_{2}\right)$ denote the subspace of all compact operators on $\ell_{2}$ (hence an operator space in a natural manner). If $1 \leq p<\infty$, then the Schatten class $S_{p}$ is defined to be the space of all compact operators $T$ on $\ell_{2}$ for which $\operatorname{tr}(|T|)^{p}<\infty$ equipped with the norm

$$
\|T\|_{S_{p}}=\left(\operatorname{tr}\left(|T|^{p}\right)\right)^{1 / p} \quad \text { for all } T \in S_{p} .
$$

If $n \in \mathbb{N}$ and $p$ is as above, $S_{p}^{n}$ denotes the space of all operators on $\ell_{2}^{n}$ equipped with the norm defined in (0.1). If also $m \in \mathbb{N}$, then $S_{p}^{n, m}$ denotes the subspace of $S_{p}$ consisting of those elements which correspond to matrices $\left(a_{i j}\right)$ where $a_{i j}=0$ unless $i \leq n$ and $j \leq m$.

From trace duality it easily follows that $S_{\infty}^{*}=S_{1}$ and hence as a dual space $S_{1}$ has a natural operator structure as defined above. It is well known that $S_{p}$ can be obtained by complex interpolation,

$$
S_{p}=\left[S_{\infty}, S_{1}\right]_{1 / p}
$$

Pisier proved in [23] that

$$
M_{n}\left(S_{p}\right)=\left[M_{n}\left(S_{\infty}\right), M_{n}\left(S_{1}\right)\right]_{1 / p}
$$


defines matrix norms on $S_{p}$ which satisfy Ruan's matrix norm characterization of operator spaces, and this is called the natural operator space structure of $S_{p}$ which we shall always use in the following.

Let $e_{i j}$ denote the element of $B\left(\ell_{2}\right)$ corresponding to the matrix with coefficients equal to one at the $i, j$ entry and zero elsewhere. If $1 \leq p \leq \infty$, we define the operator subspaces $C_{p}$ and $R_{p}$ of $S_{p}$ by

$$
C_{p}=\overline{\operatorname{span}}\left\{e_{i 1} \mid i \in \mathbb{N}\right\}, \quad R_{p}=\overline{\operatorname{span}}\left\{e_{1 j} \mid j \in \mathbb{N}\right\} .
$$

As Banach spaces, they are both isometric to $\ell_{2}$, but it follows from Pisier [23] that they are not cb-isomorphic as operator spaces.

If $1 \leq p \leq \infty$, then we put $\mathcal{K}_{p}=\left(\sum_{n=1}^{\infty} S_{p}^{n}\right)_{p} ; \mathcal{K}_{p}$ is clearly an operator space in a canonical manner.

If $H$ is an operator Hilbert space, i.e. an operator space which as a Banach space is isometric to a Hilbert space, then we put $H^{c}=C B(\mathbb{C}, H)$ and $H^{r}=C B(H, \mathbb{C})$ and if $1<p<\infty$, then we let $H^{c_{p}}=\left[H^{c}, H^{r}\right]_{1 / p}$ and $H^{r_{p}}=\left[H^{r}, H^{c}\right]_{1 / p}$.

If $E$ is an operator space and $1 \leq p \leq \infty$, it is possible to define $S_{p}[E]$ $\left(S_{p}\right.$ with values in $E$ ) as the completion of $S_{p} \otimes E$ under a certain operator space norm; we refer to [23, Chapter 1] for the details. In particular, we shall often use the following proposition proved by Pisier [23, Lemma 1.7, see also Propositions 2.3, 2.4 and Remark 2.5].

Proposition 0.1. Let $E$ and $F$ be operator spaces. A linear map $T$ : $E \rightarrow F$ is cb-bounded if and only if $\sup _{n \in \mathbb{N}}\left\|\operatorname{id}_{S_{p}^{n}} \otimes T: S_{p}^{n}[E] \rightarrow S_{p}^{n}[F]\right\|<\infty$. In that case we have $\|T\|_{\mathrm{cb}}=\sup _{n \in \mathbb{N}}\left\|\operatorname{id}_{S_{p}^{n}} \otimes T\right\|$.

The norms in $S_{p}\left[R_{p}\right]$ and $S_{p}\left[C_{p}\right]$ were computed by Pisier in [23, p. 108], and since we are going to use this frequently, we state it in a proposition.

Proposition 0.2. If $\left(x_{k}\right)_{k=1}^{n} \subseteq S_{p}$, then

$$
\left\|\sum_{k=1}^{n} x_{k} \otimes e_{1 k}\right\|_{S_{p}\left[R_{p}\right]}=\left\|\left(\sum_{k=1}^{n} x_{k} x_{k}^{*}\right)^{1 / 2}\right\|_{S_{p}}
$$

and

$$
\left\|\sum_{k=1}^{n} x_{k} \otimes e_{k 1}\right\|_{S_{p}\left[C_{p}\right]}=\left\|\left(\sum_{k=1}^{n} x_{k}^{*} x_{k}\right)^{1 / 2}\right\|_{S_{p}} .
$$

If $X$ is a subspace of $S_{p}$ and $E$ is an operator space, then we let $X[E]$ denote the closure of $E \otimes X$ in $S_{p}[E]$.

Let $A$ be a von Neumann algebra with a normal semifinite faithful trace $\tau$ (i.e. $A$ is semifinite). The ideal

$$
m(\tau)=\left\{\sum_{k=1}^{n} x_{k} y_{k} \mid n \in \mathbb{N}, \sum_{k=1}^{n}\left[\tau\left(y_{k}^{*} y_{k}\right)+\tau\left(x_{k}^{*} x_{k}\right)\right]<\infty\right\}
$$


is called the definition ideal of $\tau$ on which there is a unique linear extension $\tau: m(\tau) \rightarrow \mathbb{C}$ so that $\tau(x y)=\tau(y x)$ for all $x, y \in m(\tau)$ (see e.g. [28]). If $1 \leq p<\infty$, then we put

$$
\|x\|=\tau\left(\left(x^{*} x\right)^{p / 2}\right)^{1 / p} \quad \text { for all } x \in m(\tau),
$$

which is readily seen to be a norm on $m(\tau)$. We define $L_{p}(A, \tau)$ to be the completion of $m(\tau)$ under this norm. Conventionally we put $L_{\infty}(A, \tau)=A$. It follows easily that $L_{1}(A, \tau)^{*}=A^{\text {op }}$, where $A^{\text {op }}$ denotes $A$ equipped with the reverse (or opposite) multiplication, and hence $L_{1}(A, \tau)$ has a natural operator space structure. It can be shown that the complex interpolation method yields

$$
L_{p}(A, \tau)=\left[A, L_{1}(A, \tau)\right]_{1 / p} .
$$

Pisier [23] proved that

$$
M_{n}\left(L_{p}(A, \tau)\right)=\left[M_{n}(A), M_{n}\left(L_{1}(A, \tau)\right)\right]_{1 / p}
$$

defines a natural operator space structure on $L_{p}(A, \tau)$, which we shall use in what follows. If $\tau_{1}$ is another normal semifinite faithful trace on $A$, then it can easily be shown that $L_{p}(A, \tau)$ is cb-isometric to $L_{p}\left(A, \tau_{1}\right)$, and therefore we shall often write $L_{p}(A)$ instead of $L_{p}(A, \tau)$.

If $B$ is a von Neumann subalgebra of $A$ so that the restriction of $\tau$ to $B$ is semifinite again, then it follows from [28, Proposition 2.36] that there exists a faithful normal projection $E_{B}$ of $A$ onto $B$ such that $\tau=\tau \circ E_{B}$. The projection $E_{B}$ is called the conditional expectation of $A$ onto $B$.

An operator space $X$ is called an operator $\mathcal{L}_{p^{-}}$space (for short, $\mathcal{O} \mathcal{L}_{p^{-}}$ space), $1 \leq p \leq \infty$, if there exist a $\lambda \geq 1$ and a cofinal family $\left(F_{j}\right)_{j \in I}$ of finite-dimensional subspaces such that $\bigcup_{j \in I} F_{j}$ is dense in $X$ and for every $j$ there exists a finite-dimensional $C^{*}$-algebra $A_{j}$ with

$$
d_{\mathrm{cb}}\left(L_{p}\left(A_{j}\right), F_{j}\right) \leq \lambda .
$$

In this case we shall also say that $X$ is an $\mathcal{O} \mathcal{L}_{p, \lambda^{-}}$space. $X$ is called an $\mathcal{O} \mathcal{S}_{p, \lambda}$-space if we can replace the $L_{p}\left(A_{j}\right)$ 's in $(0.4)$ by $S_{p}^{n_{j}}$ 's, and a completely complemented $\mathcal{O} \mathcal{L}_{p, \lambda}$-space (for short, $\mathcal{C O} \mathcal{L}_{p, \lambda}$-space) if in addition the $F_{j}$ 's can be chosen to be cb-complemented in $X$ by projections with cb-norms less than or equal to $\lambda . \mathcal{C O} \mathcal{S}_{p, \lambda}$-spaces are defined similarly.

If the $L_{p}\left(A_{j}\right)$ 's in $(0.4)$ are of the form $\left(\bigoplus_{i=1}^{k} S_{p}^{n(i), m(i)}\right)_{p}$, then $X$ is called a rectangular $\mathcal{O} \mathcal{L}_{p}$-space.

Let $1 \leq p \leq \infty$. An operator space $X$ is said to have the $\gamma_{p}$-approximation property (for short, $\gamma_{p}$-AP) if there exists a $\lambda>0$ and nets $\left(U_{i}\right)$ and $\left(V_{i}\right)$ of finite rank operators, $U_{i}: X \rightarrow S_{p}, V_{i}: S_{p} \rightarrow X$, so that $\left\|U_{i}\right\|_{\mathrm{cb}}\left\|V_{i}\right\|_{\mathrm{cb}} \leq \lambda$ and $\left(V_{i} U_{i}\right)$ converges pointwise to the identity of $X$.

Finally, if $\left(x_{n}\right)$ is a finite or infinite sequence in a Banach space $X$, we let $\left[x_{n}\right]$ denote the closed linear span of the sequence $\left(x_{n}\right)$. If $A$ is a set, $|A|$ 
denotes its cardinality, and if $X$ and $Y$ are Banach spaces, $X \oplus_{p} Y$ stands for the direct sum of $X$ and $Y$ equipped with the norm $\left(\|\cdot\|_{X}^{p}+\|\cdot\|_{Y}^{p}\right)^{1 / p}$.

1. The Rosenthal operator spaces and their basic properties. In this section we shall investigate some operator spaces which correspond to the $\mathcal{L}_{p^{-}}$-spaces in Banach space theory constructed by Rosenthal in [26].

We let $2<p<\infty, 1 / p+1 / p^{\prime}=1,1 / 2=1 / p+1 / r($ i.e. $r=2 p /(p-2))$ and let $\sigma=\left(\sigma_{n}\right)$ be a sequence of real numbers with $\sigma_{n}>0$ for all $n \in \mathbb{N}$. We denote the unit vector basis of $\ell_{2}$ by $\left(\xi_{n}\right)$ and let $D_{\sigma}$ be the diagonal operator on $\ell_{2}$ defined by $D_{\sigma} \xi_{n}=\sigma_{n} \xi_{n}$ for all $n \in \mathbb{N}$.

Our first space $\widetilde{X}_{p}(\sigma)$ is defined to be the space of all sequences $a=\left(a_{n}\right)$ which satisfy

$$
\sum_{n=1}^{\infty}\left|a_{n}\right|^{p}<\infty \text { and } \sum_{n=1}^{\infty}\left|a_{n}\right|^{2} \sigma_{n}^{2}<\infty,
$$

equipped with the norm

$$
\|a\|=\left(\sum_{n=1}^{\infty}\left|a_{n}\right|^{p}+\left(\sum_{n=1}^{\infty}\left|a_{n}\right|^{2} \sigma_{n}^{2}\right)^{p / 2}\right)^{1 / p} .
$$

$\widetilde{X}_{p}(\sigma)$ is the classical Rosenthal sequence space (except that he used an equivalent norm) and we can clearly identify it with the closed linear span in $S_{p} \oplus_{p} S_{2}$ of the sequence $\left\{\left(e_{n n}, \sigma_{n} e_{n n}\right) \mid n \in \mathbb{N}\right\}$. As an operator space we can however represent $\widetilde{X}_{p}(\sigma)$ in three different ways. We define $X_{p, c_{p}}(\sigma)$ to be the closed linear span of $\left\{\left(e_{n n}, \sigma_{n} e_{n 1}\right) \mid n \in \mathbb{N}\right\}$ in $S_{p} \oplus C_{p}$. Similarly we let $X_{p, r_{p}}(\sigma)$ denote the closed linear span of $\left\{\left(e_{n n}, \sigma_{n} e_{1 n}\right) \mid n \in \mathbb{N}\right\}$ in $S_{p} \oplus R_{p}$, and finally we let $X_{p}(\sigma)$ denote the closed linear span of $\left\{\left(e_{n n}, \sigma_{n} e_{n 1}, \sigma_{n} e_{1 n}\right)\right\}$ in $S_{p} \oplus C_{p} \oplus R_{p}$.

Since $S_{p} \oplus C_{p} \oplus R_{p}$ is cb-isomorphic to $S_{p}$, each of the above three spaces is cb-isomorphic to a subspace of $S_{p}$. We shall often let $X_{p *}(\sigma)$ denote any of them.

Since we shall often use Proposition 0.1 to check cb-boundedness, it is worth mentioning how the norms in $S_{p}\left[X_{p, r_{p}}(\sigma)\right], S_{p}\left[X_{p, c_{p}}(\sigma)\right]$, and $S_{p}\left[X_{p}(\sigma)\right]$ can be computed. It follows immediately from Proposition 0.2 that if $\left(x_{k}\right)_{k=1}^{n}$ $\subseteq S_{p}$, then

$$
\begin{aligned}
\| \sum_{k=1}^{n} x_{k} \otimes\left(e_{k k} \oplus \sigma_{k} e_{1 k}\right) & \|_{S_{p}\left[X_{p, r_{p}}(\sigma)\right]} \\
= & \left(\sum_{k=1}^{n}\left\|x_{k}\right\|^{p}+\left\|\left(\sum_{k=1}^{n} \sigma_{k}^{2} x_{k} x_{k}^{*}\right)^{1 / 2}\right\|_{S_{p}}^{p}\right)^{1 / p},
\end{aligned}
$$




$$
\begin{aligned}
\| \sum_{k=1}^{n} x_{k} \otimes\left(e_{k k} \oplus \sigma_{k} e_{k 1}\right) & \|_{S_{p}\left[X_{p, c_{p}}(\sigma)\right]} \\
= & \left(\sum_{k=1}^{n}\left\|x_{k}\right\|^{p}+\left\|\left(\sum_{k=1}^{n} \sigma_{k}^{2} x_{k}^{*} x_{k}\right)^{1 / 2}\right\|_{S_{p}}^{p}\right)^{1 / p},
\end{aligned}
$$

and similarly for $S_{p}\left[X_{p}(\sigma)\right]$.

It follows easily from these formulas and Proposition 0.1 that though isometric as Banach spaces, these three spaces are not mutually cb-isomorphic as operator spaces.

Throughout the paper we shall often impose at least one of the following two conditions on $\sigma$ :

$$
\begin{gathered}
\liminf _{n \rightarrow \infty} \sigma_{n}=0, \\
\sum_{\sigma_{n} \leq \varepsilon} \sigma_{n}^{r}=\infty \quad \text { for all } \varepsilon>0 .
\end{gathered}
$$

It is immediate that if $\sigma_{n} \rightarrow 0$ and $\sigma \notin \ell_{r}$, then (1.5) and (1.6) are satisfied. (1.6) ensures that the operator $x \mapsto x D_{\sigma}$ does not act as a bounded operator from $S_{p}$ to $S_{2}$.

It follows from [26] that $\widetilde{X}_{p}(\sigma)$ is an $\mathcal{L}_{p^{-}}$space if and only if $(1.5)$ is satisfied, and if both (1.5) and (1.6) hold, then $\widetilde{X}_{p}(\sigma)$ is the classical Rosenthal $\mathcal{L}_{p^{-}}$-space which is unique up to a Banach space isomorphism. Later in this section we shall prove a similar uniqueness result for the operator space versions.

Our first result states:

Theorem 1.1. If $\sigma$ satisfies (1.5) and (1.6), then $\widetilde{X}_{p}(\sigma)^{*}$ is not Banach space isomorphic to a subspace of $S_{p^{\prime}}$. Consequently, $\widetilde{X}_{p}(\sigma)$ is not Banach space isomorphic to a complemented subspace of $S_{p}$.

Proof. Assume that $\widetilde{X}_{p}(\sigma)^{*}$ is isomorphic to a subspace of $S_{p^{\prime}}$ and fix $n \in \mathbb{N}$. By [26, Corollary 8], $\widetilde{X}_{p}(\sigma)^{*}$ contains a basic sequence $\left(h_{k}\right)$ equivalent to the unit vector basis of $\ell_{2}$ such that the set of any $n$ elements of that sequence is isometrically equivalent to the unit vector basis of $\ell_{p^{\prime}}^{n}$. From [2, Proposition 4 and Lemma 1] it follows that $\left(h_{k}\right)$ has a subsequence which is 4 -equivalent to the unit vector basis of $\ell_{2}$. This is a contradiction for large $n \in \mathbb{N}$.

The next theorem is the operator space version of Rosenthal's Lemma 7 in $[26]$.

Proposition 1.2. Let $\left(g_{n}\right)$ be the natural basis of $X_{p *}(\sigma)$ and let $\left(E_{j}\right)$ be a sequence of mutually disjoint finite subsets of $\mathbb{N}$. For each $j \in \mathbb{N}$ put 


$$
\begin{aligned}
& f_{j}=\sum_{n \in E_{j}} \sigma_{n}^{r / p} g_{n}, \\
& \beta_{j}=\left(\sum_{n \in E_{j}} \sigma_{n}^{r}\right)^{1 / r}, \\
& \tilde{f}_{j}=\beta_{j}^{-r / p} f_{j} .
\end{aligned}
$$

Then $\left(\widetilde{f}_{j}\right)$ is a cb-unconditional basic sequence, cb-isometrically equivalent to the natural basis of $X_{p *}(\beta)$, and there is a cb-contractive projection of $X_{p *}$ onto $\left[f_{j}\right]$.

Proof. We shall prove the theorem for $X_{p, c_{p}}(\sigma)$; the other cases can be proved in a similar manner.

If $\left(x_{j}\right)_{j=1}^{k} \subseteq S_{p}$, then we get

$$
\left\|\sum_{j=1}^{k} x_{j} \otimes f_{j}\right\|_{S_{p}\left[X_{p, c_{p}}(\sigma)\right]}=\left\|\sum_{j=1}^{k} \sum_{n \in E_{j}} \sigma_{n}^{r / p} x_{j} \otimes\left[e_{n n} \oplus \sigma_{n} e_{n 1}\right]\right\|_{S_{p}\left[X_{p, c_{p}}(\sigma)\right]} .
$$

It easily follows that

$$
\left\|\sum_{j=1}^{k} \sum_{n \in E_{j}} \sigma_{n}^{r / p} x_{j} \otimes e_{n n}\right\|_{S_{p}\left[S_{p}\right]}=\left(\sum_{j=1}^{k}\left\|x_{j}\right\|^{p} \sum_{n \in E_{j}} \sigma_{n}^{r}\right)^{1 / p}=\left(\sum_{j=1}^{k}\left\|x_{k}\right\|^{p} \beta_{j}^{r}\right)^{1 / p} .
$$

From (0.3) we get

$$
\begin{aligned}
\left\|\sum_{j=1}^{k} \sum_{n \in E_{j}} \sigma_{n}^{r / p} x_{j} \otimes \sigma_{n} e_{n 1}\right\|_{S_{p}\left[C_{p}\right]} & =\left\|\left(\sum_{j=1}^{k} \sum_{n \in E_{j}} \sigma_{n}^{2 r / p+2} x_{j}^{*} x_{j}\right)^{1 / 2}\right\|_{S_{p}} \\
& =\left\|\left(\sum_{j=1}^{k} \beta_{j}^{r} x_{j}^{*} x_{j}\right)^{1 / 2}\right\|_{S_{p}}
\end{aligned}
$$

and therefore

$$
\left\|\sum_{j=1}^{k} x_{j} \otimes \tilde{f}_{j}\right\|_{S_{p}\left[X_{p, c_{p}}(\sigma)\right]}=\left\|\sum_{j=1}^{k} x_{j} \otimes\left[e_{j j} \oplus \beta_{j} e_{j 1}\right]\right\|_{S_{p}\left[X_{p, c_{p}}(\beta)\right]} .
$$

Together with Proposition 0.1 this shows that $\left(\tilde{f}_{j}\right)$ is cb-isometrically equivalent to the natural basis $\left(g_{j}\right)$ of $X_{p, c_{p}}(\beta)$.

For all $x, y \in X_{p, c_{p}}(\sigma)$ we put $\langle x, y\rangle=\sum_{j=1}^{\infty} x(j) \overline{y(j)} \sigma_{j}^{2}$ (where $x(j)$, respectively $y(j)$, denotes the $j$ th coordinate of $x$, respectively $y$, in the basis $\left.\left(g_{j}\right)\right)$ and define

$$
P x=\sum_{j=1}^{\infty}\left\langle x, f_{j}\right\rangle \beta^{-r} f_{j} \quad \text { for all } x \in X_{p, c_{p}}(\sigma) .
$$


It follows immediately from Rosenthal's argument in [26, Lemma 7] that in the Banach space sense $P$ is a contractive projection of $X_{p, c_{p}}(\sigma)$ onto $\left[f_{j}\right]$. In addition we need to prove that $P$ is completely bounded with $\|P\|_{\mathrm{cb}}=1$.

For every $n \in \mathbb{N}$ we get

$$
\begin{aligned}
P g_{n} & =\sum_{j=1}^{\infty}\left\langle g_{n}, f_{j}\right\rangle \beta_{j}^{-r} f_{j}=\sigma_{n}^{r / p+2} \beta_{j_{n}} f_{j_{n}} \\
& =\sigma_{n}^{r / p+2} \beta_{j_{n}}^{r / p-r} \widetilde{f}_{j_{n}}=\beta_{j_{n}}^{-r / p^{\prime}} \sigma_{n}^{r / p^{\prime}} \widetilde{f}_{j_{n}},
\end{aligned}
$$

where $j_{n}$ is chosen such that $n \in E_{j_{n}}$.

Let now $\left(x_{n}\right) \subseteq S_{p}$ be a finite sequence. From (1.12) and the first part of the proof we obtain

$$
\begin{aligned}
\| \sum_{n} x_{n} & \otimes P g_{n} \|_{S_{p}\left[X_{p, c_{p}}\right]} \\
= & \left\|\sum_{j} \beta_{j}^{-r / p^{\prime}}\left(\sum_{n \in E_{j}} \sigma_{n}^{r / p^{\prime}} x_{n}\right) \otimes \widetilde{f}_{j}\right\|_{S_{p}\left[X_{p, c_{p}}(\sigma)\right]} \\
= & \left\|\sum_{j} \beta_{j}^{-r / p^{\prime}}\left(\sum_{n \in E_{j}} \sigma_{n}^{r / p^{\prime}} x_{n}\right) \otimes\left[e_{j j} \oplus \beta_{j} e_{j 1}\right]\right\|_{S_{p}\left[X_{p, c_{p}}(\beta)\right]} .
\end{aligned}
$$

We estimate the two coordinates separately and start with

$$
\begin{aligned}
\left\|\sum_{j} \beta_{j}^{-r / p^{\prime}}\left(\sum_{n \in E_{j}} \sigma_{n}^{r / p^{\prime}} x_{n}\right) \otimes e_{j j}\right\|_{S_{p}\left[S_{p}\right]} & \left(\sum_{j} \beta_{j}^{-r p / p^{\prime}}\left\|\sum_{n \in E_{j}} \sigma_{n}^{r / p^{\prime}} x_{n}\right\|_{S_{p}}^{p}\right)^{1 / p} \\
\leq & \left(\sum_{j} \beta_{j}^{-r p / p^{\prime}}\left(\sum_{n \in E_{j}} \sigma_{n}^{r}\right)^{p / p^{\prime}} \sum_{n \in E_{j}}\left\|x_{n}\right\|_{S_{p}}^{p}\right)^{1 / p} \\
& =\left(\sum_{j} \sum_{n \in E_{j}}\left\|x_{n}\right\|_{S_{p}}^{p}\right)^{1 / p}=\left(\sum_{n}\left\|x_{n}\right\|_{S_{p}}^{p}\right)^{1 / p} .
\end{aligned}
$$

The estimate of the other coordinate is slightly more involved. For every $\xi \in \ell_{2}$ and every $j$ we get

$$
\begin{aligned}
\left(\left(\sum_{n \in E_{j}} \sigma_{n}^{r / p^{\prime}} x_{n}^{*}\right)\left(\sum_{n \in E_{j}} \sigma_{n}^{r / p^{\prime}} x_{n}\right) \xi, \xi\right) & \\
= & \left\|\sum_{n \in E_{j}} \sigma_{n}^{r / p^{\prime}} x_{n} \xi\right\|^{2} \leq\left(\sum_{n \in E_{j}} \sigma_{n}^{2 r / p^{\prime}-2}\right)\left(\sum_{n \in E_{j}}\left\|\sigma_{n} x_{n} \xi\right\|^{2}\right) \\
& =\sum_{n \in E_{j}} \sigma_{n}^{r} \sum_{n \in E_{j}}\left(\sigma_{n}^{2} x_{n}^{*} x_{n} \xi, \xi\right)=\beta_{j}^{r} \sum_{n \in E_{j}} \sigma_{n}^{2}\left(x_{n}^{*} x_{n} \xi, \xi\right),
\end{aligned}
$$


which shows that in the sense of operators on $\ell_{2}$ we have

$$
0 \leq \sum_{j} \beta_{j}^{-r}\left(\sum_{n \in E_{j}} \sigma_{n}^{r / p^{\prime}} x_{n}^{*}\right)\left(\sum_{n \in E_{j}} \sigma_{n}^{r / p^{\prime}} x_{n}\right) \leq \sum_{j} \sigma_{j}^{2} x_{j}^{*} x_{j} .
$$

Together with (0.3) and [7, Theorem 2.3] this gives

$$
\begin{aligned}
\left\|\sum_{j} \beta_{j}^{-r / p^{\prime}} \sum_{n \in E_{j}} \sigma_{n}^{r / p^{\prime}} x_{n} \otimes \beta_{j} e_{j 1}\right\|_{S_{p}\left[C_{p}\right]} \\
=\left\|\left(\sum_{j} \beta_{j}^{-r}\left(\sum_{n \in E_{j}} \sigma_{n}^{r / p^{\prime}} x_{n}^{*}\right)\left(\sum_{n \in E_{j}} \sigma_{n}^{r / p^{\prime}} x_{n}\right)\right)^{1 / 2}\right\|_{S_{p}} \\
=\left(\operatorname{tr}\left(\left[\sum_{j} \beta_{j}^{-r} \sum_{n \in E_{j}} \sigma_{n}^{r / p^{\prime}} x_{n}^{*} \sum_{n \in E_{j}} \sigma_{n}^{r / p} x_{n}\right]^{p / 2}\right)\right)^{1 / p} \\
\leq\left(\operatorname{tr}\left(\left[\sum_{j} \sigma_{j}^{2} x_{j}^{*} x_{j}\right]^{p / 2}\right)\right)^{1 / p}=\left\|\sum_{j} x_{j} \otimes \sigma_{j} e_{j 1}\right\|_{S_{p}\left[C_{p}\right]} .
\end{aligned}
$$

(1.13)-(1.15) show that $P$ is completely bounded with $\|P\|_{\mathrm{cb}}=1$.

An application of Theorem 1.1 shows, as in the Banach space case, that if $\sigma$ in addition satisfies (1.6), then $X_{p *}(\sigma)$ is uniquely determined up to a cb-isomorphism. This is the contents of the next theorem.

TheOREM 1.3. If $2<p<\infty$, and $\sigma$ and $\gamma$ are two sequences both satisfying (1.5) and (1.6), then $X_{p *}(\sigma)$ is cb-isomorphic to $X_{p *}(\gamma)$.

Proof. The proof follows the lines of the proofs of [26, Proposition 12 and Theorem 13] and is based on Pełczyński's decomposition method (see e.g. [18, Theorem 2.a.3]). We will therefore first prove that $X_{p *}(\gamma)$ is cb-isomorphic to a cb-complemented subspace of $X_{p *}(\sigma)$ and vice versa.

Since $\sigma$ satisfies (1.5) and (1.6), we can find a sequence $\left(E_{j}\right)$ of mutually disjoint, finite subsets of $\mathbb{N}$ so that

$$
\gamma_{j} \leq \beta_{j}=\left(\sum_{n \in E_{j}} \sigma_{n}^{r}\right)^{1 / r} \leq 2 \gamma_{j} \quad \text { for all } j \in \mathbb{N} .
$$

From Proposition 1.2 it follows that $X_{p *}(\beta)$ is cb-isometric to a subspace of $X_{p *}(\sigma)$ onto which there is a cb-contractive projection. (1.16) shows that $X_{p *}(\gamma)$ is 2-cb-isomorphic to $X_{p *}(\beta)$. By interchanging the roles of $\gamma$ and $\sigma$ we find that also $X_{p *}(\sigma)$ is cb-isomorphic to a cb-complemented subspace of $X_{p *}(\gamma)$.

The next step is to show that $X_{p *}(\sigma)$ is cb-isomorphic to $X_{p *}(\sigma) \oplus X_{p *}(\sigma)$, but we shall only prove it for $X_{p, c_{p}}(\sigma)$ since the other cases can be obtained in a similar manner. 
(1.5) and (1.6) imply that we can find a sequence $\left\{E_{j, k} \mid j \in \mathbb{N}, k \in \mathbb{N}\right\}$ of mutually disjoint finite subsets of $\mathbb{N}$ so that

$$
\sigma_{j} \leq \beta_{j, k}=\left(\sum_{n \in E_{j, k}} \sigma_{n}^{r}\right)^{1 / r} \leq 2 \sigma_{j} \quad \text { for all } j, k \in \mathbb{N} .
$$

Put $\beta_{k}=\left(\beta_{j, k}\right)_{j=1}^{\infty}$, let $\tilde{f}_{j, k}=\beta_{j, k}^{-r / p} \sum_{n \in E_{j, k}} \sigma_{n}^{r / p} e_{n n} \oplus \sigma_{n} e_{n 1}$ and define $Z=\left[\widetilde{f}_{j, k} \mid j, k \in \mathbb{N}\right], Z_{1}=\left[\widetilde{f}_{j, k} \mid j \in \mathbb{N}, k \geq 2\right]$. It follows from Proposition 1.2 that $Z$ is cb-contractively complemented in $X_{p, c_{p}}(\sigma)$ and that for all $k \in \mathbb{N},\left[\widetilde{f}_{j, k}\right]$ is cb-contractively complemented and cb-isometric to $X_{p, c_{p}}\left(\left(\beta_{k}\right)\right)$, which in turn is 2-cb-isomorphic to $X_{p, c_{p}}(\sigma)$. Hence $Z$ can be viewed as an infinite direct sum of copies of $X_{p, c_{p}}(\sigma)$. Let $T: \operatorname{span}\left\{\tilde{f}_{j, k} \mid\right.$ $j, k \in \mathbb{N}\} \rightarrow Z_{1}$ be defined by $T \widetilde{f}_{j, k}=\widetilde{f}_{j, k+1}$. We shall show that $T$ extends to a cb-isomorphism of $Z$ onto $Z_{1}$. If $\left(x_{j, k}\right) \subseteq S_{p}$ is a finite sequence, then we deduce from (1.17) and [7, Theorem 2.3] that

$$
\begin{aligned}
\left\|\left(\sum_{k} \sum_{j} \beta_{j, k+1}^{2} x_{j, k}^{*} x_{j, k}\right)^{1 / 2}\right\|_{S_{p}} & \leq 2\left\|\left(\sum_{k} \sum_{j} \sigma_{j}^{2} x_{j, k}^{*} x_{j, k}\right)^{1 / 2}\right\|_{S_{p}} \\
& \leq 2\left\|\left(\sum_{k} \sum_{j} \beta_{j, k}^{2} x_{j, k}^{*} x_{j, k}\right)^{1 / 2}\right\|_{S_{p}} .
\end{aligned}
$$

In the same manner we get

$$
\left\|\left(\sum_{k} \sum_{j} \beta_{j, k}^{2} x_{j, k}^{*} x_{j, k}\right)^{1 / 2}\right\|_{S_{p}} \leq 2\left\|\left(\sum_{k} \sum_{j} \beta_{j, k+1} x_{j, k}^{*} x_{j, k}\right)^{1 / 2}\right\| .
$$

Similar estimates can easily be obtained for the corresponding $p$-norms, which implies that

$$
\begin{aligned}
\frac{1}{2}\left\|\sum_{k} \sum_{j} x_{j, k} \otimes \widetilde{f}_{j, k}\right\|_{S_{p}\left[X_{p, c_{p}}(\sigma)\right]} & \leq\left\|\sum_{k} \sum_{j} x_{j, k} \otimes \widetilde{f}_{j, k+1}\right\|_{S_{p}\left[X_{p, c_{p}}(\sigma)\right]} \\
& \leq 2\left\|\sum_{k} \sum_{j} x_{j, k} \otimes \tilde{f}_{j, k}\right\|_{S_{p}\left[X_{p, c_{p}}(\sigma)\right]}
\end{aligned}
$$

showing that $T$ can be extended to a cb-isomorphism of $Z$ onto $Z_{1}$.

Letting $\sim_{\mathrm{cb}}$ denote "cb-isomorphic to", we infer from the above that $Z \sim_{\text {cb }} X_{p, c_{p}}(\sigma) \oplus Z$. Since $Z$ is cb-complemented in $X_{p, c_{p}}(\sigma)$, we can find a closed subspace $U \subseteq X_{p, c_{p}}(\sigma)$ such that

$$
X_{p, c_{p}}(\sigma)=Z \oplus U \sim_{\mathrm{cb}} X_{p, c_{p}}(\sigma) \oplus Z \oplus U \sim_{\mathrm{cb}} X_{p, c_{p}}(\sigma) \oplus X_{p, c_{p}}(\sigma) .
$$

We are now ready to show that $X_{p, c_{p}}(\gamma)$ is cb-isomorphic to $X_{p, c_{p}}(\sigma)$. Indeed, since by the above $X_{p, c_{p}}(\gamma)$ is cb-isomorphic to a cb-complemented subspace of $X_{p, c_{p}}(\sigma)$, we can find a closed subspace $G \subseteq X_{p, c_{p}}(\sigma)$ such that 


$$
\begin{aligned}
X_{p, c_{p}}(\sigma) & \sim_{\mathrm{cb}} X_{p, c_{p}}(\gamma) \oplus G \sim_{\mathrm{cb}} X_{p, c_{p}}(\gamma) \oplus X_{p, c_{p}}(\gamma) \oplus G \\
& \sim_{\mathrm{cb}} X_{p, c_{p}}(\gamma) \oplus X_{p, c_{p}}(\sigma) \sim_{\mathrm{cb}} X_{p, c_{p}}(\gamma)
\end{aligned}
$$

where the last $\sim_{\mathrm{cb}}$ follows by interchanging the roles of $\sigma$ and $\gamma$.

Exploiting the decomposition method a bit more we can actually conclude that also the space $Z$ in the above proof is cb-isomorphic to $X_{p, c_{p}}(\sigma)$.

We are now going to define some operator spaces which we shall call matricial Rosenthal spaces.

Define $\widetilde{Y}_{p}(\sigma)$ to be the subspace of $\mathcal{K}_{p} \oplus_{p}\left(\sum_{n=1}^{\infty} S_{2}^{n}\right)_{2}$ consisting of all elements of the form $\left(\left(x_{n}, \sigma_{n} x_{n}\right)\right)$ where $x_{n} \in S_{p}^{n}$ for all $n \in \mathbb{N}$, i.e. we require

$$
\sum_{n=1}^{\infty}\left\|x_{n}\right\|_{S_{p}^{n}}^{p}<\infty \text { and } \sum_{n=1}^{\infty} \sigma_{n}^{2}\left\|x_{n}\right\|_{S_{2}^{n}}^{2}<\infty .
$$

We can view $\left(\sum_{n=1}^{\infty} S_{2}^{n}\right)_{2}$ isometrically as a subspace of $C_{p}\left[C_{p}\right]$ in the following way: Choose a sequence $\left(m_{n}\right)$ of integers so that $m_{1}=0$ and $m_{n+1}-m_{n}$ $=n$ for all $n \in \mathbb{N}$. If $x=\left(x_{n}\right) \in\left(\sum_{n=1}^{\infty} S_{2}^{n}\right)_{2}$ with $x_{n}=\left(t_{i j}^{n}\right)_{i, j=1}^{n}$, we can identify $x$ with $\sum_{n=1}^{\infty} \sum_{i, j=m_{n}+1}^{m_{n+1}} t_{i j}^{n} e_{i j} \in C_{p}\left[C_{p}\right]$. Similarly we can consider $\left(\sum_{n=1}^{\infty} S_{2}^{n}\right)_{2}$ as a subspace of $R_{p}\left[R_{p}\right]$, respectively of $C_{p}\left[C_{p}\right] \oplus_{p} R_{p}\left[R_{p}\right]$.

Hence there is a canonical Banach space isometry $w_{\sigma}$ of $\widetilde{Y}_{p}(\sigma)$ into the operator space $\mathcal{K}_{p} \oplus_{p} C_{p}\left[C_{p}\right]$ and we put $Y_{p, c_{p}}=w_{\sigma}\left(\widetilde{Y}_{p}(\sigma)\right)$. Similarly we define the spaces $Y_{p, r_{p}}(\sigma)$ and $Y_{p, c_{p} \cap r_{p}}(\sigma)$. In the rest of this paper we shall put $Y_{p}(\sigma)=Y_{p, c_{p} \cap r_{p}}(\sigma)$.

Since we often consider cb-maps to or from these spaces, it is worth mentioning how the norm in $S_{p}\left[Y_{p, c_{p}}(\sigma)\right]$ is computed (the other cases follow similarly). Let us just compute the "column part" of $S_{p}\left[Y_{p, c_{p}}(\sigma)\right]$. To this end let $X_{n} \in S_{p} \otimes S_{p}^{n}$ for all $n \in \mathbb{N}$. We can then find $\left(x_{j k}^{n}\right) \in S_{p}^{n}$ so that

$$
X_{n}=\sum_{j, k=m_{n}+1}^{m_{n+1}} x_{j k}^{n} \otimes e_{j k}
$$

for every $n \in \mathbb{N}$. Note that

$$
X_{n}^{*} X_{n}=\sum_{k, l=m_{n}+1}^{m_{n+1}}\left(\sum_{j=m_{n}+1}^{m_{n+1}} x_{j k}^{n *} x_{j l}^{n}\right) e_{k l} .
$$

Using Proposition 0.2 we get

$$
\left\|\sum_{n} \sigma_{n} X_{n}\right\|_{S_{p}\left[C_{p}\left[C_{p}\right]\right]}=\left\|\sum_{n} \sum_{j, k=m_{n}+1}^{m_{n+1}} x_{j k}^{n} \otimes e_{j k}\right\|_{S_{p}\left[C_{p}\left[C_{p}\right]\right]}
$$

$$
=\left\|\left(\sum_{n} \sigma_{n}^{2} \sum_{j, k=m_{n}+1}^{m_{n+1}} x_{j k}^{n *} x_{j k}^{n}\right)^{1 / 2}\right\|_{S_{p}}=\left\|\left(\sum_{n} \sigma_{n}^{2}(\mathrm{id} \otimes \operatorname{tr})\left(X_{n}^{*} X_{n}\right)\right)^{1 / 2}\right\|_{S_{p}},
$$


where we have used (1.21) to get the last equality. Comparing this with the similar calculations for the other cases it is readily verified that $Y_{p}(\sigma)$, $Y_{p, c_{p}}(\sigma)$, and $Y_{p, r_{p}}(\sigma)$ are mutually non-cb-isomorphic.

Since $\mathcal{K}_{p} \oplus_{p} C_{p}\left[C_{p}\right]$ is cb-isomorphic to a subspace of $S_{p}$, the same holds for $Y_{p, c_{p}}(\sigma)$ as well. In a similar manner we show that $Y_{p, r_{p}}(\sigma)$ and $Y_{p}(\sigma)$ are cb-isomorphic to subspaces of $S_{p}$. We have the following result on these spaces.

THEOREM 1.4. Both $\mathcal{K}_{p}$ and $X_{p, c_{p}}(\sigma)$ (respectively $X_{p, r_{p}}(\sigma)$ ) are cb-isomorphic to complemented subspaces of $Y_{p, c_{p}}(\sigma)$ (respectively $Y_{p, r_{p}}(\sigma)$ ). Consequently, $\widetilde{Y}_{p}(\sigma)$ is not Banach space isomorphic to a complemented subspace of $S_{p}$ if $\sigma$ satisfies (1.5) and (1.6).

Proof. Let $U=X_{p, c_{p}}(\sigma)$ (respectively $U=X_{p, r_{p}}(\sigma)$ ) and $W=Y_{p, c_{p}}(\sigma)$ (respectively $\left.W=Y_{p, r_{p}}(\sigma)\right)$. If $\left(n_{k}\right) \subseteq \mathbb{N}$ is a sequence with $\sum_{k=1}^{\infty} \sigma_{n_{k}}^{2 p /(p-2)}$ $<\infty$, then the subspace $V$ consisting of those $\left(\left(x_{n}, \sigma_{n} x_{n}\right)\right) \in W$ for which $x_{n}=0$ for all $n \neq n_{k}$ is readily seen to be completely complemented by a projection of cb-norm 1 and completely isomorphic to $\mathcal{K}_{p}$.

It is obvious that $U$ can be identified cb-isometrically with the subspace of $W$ consisting of those $\left(\left(x_{n}, \sigma_{n} x_{n}\right)\right) \in \widetilde{Y}_{p}(\sigma)$ for which $x_{n}$ is a one-dimensional operator on $\ell_{2}$ for all $n \in \mathbb{N}$. This space is clearly the range of a cb-contractive projection.

It now follows directly from Theorem 1.1 that $\widetilde{Y}_{p}(\sigma)$ cannot be Banach space isomorphic to a complemented subspace of $S_{p}$ if $\sigma$ satisfies (1.5) and (1.6).

The last spaces we are going to investigate are defined as follows:

$$
\begin{gathered}
Z_{p, c_{p}}(\sigma)=\left\{\left(x, x D_{\sigma}\right) \mid x \in A_{\sigma}\right\} \subseteq S_{p} \oplus_{p} C_{p}\left[C_{p}\right], \\
Z_{p, r_{p}}(\sigma)=\left\{\left(x, D_{\sigma} x\right) \mid x \in A_{\sigma}\right\} \subseteq S_{p} \oplus_{p} R_{p}\left[R_{p}\right], \\
Z_{p}(\sigma)=\left\{\left(x, x D_{\sigma}, D_{\sigma} x\right) \mid x \in A_{\sigma}\right\} \subseteq S_{p} \oplus_{p} C_{p}\left[C_{p}\right] \oplus_{p} R_{p}\left[R_{p}\right],
\end{gathered}
$$

where

$$
A_{\sigma}=\left\{x \in S_{p} \mid x D_{\sigma} \in S_{2}\right\}
$$

In (1.23) we consider $x D_{\sigma}$ as an element of $C_{p}\left[C_{p}\right]$, and similarly in (1.24) and (1.25).

In what follows we let $Z_{p, *}(\sigma)$ denote any of these spaces. Clearly they are isomorphic as Banach spaces, mutually non-cb-isomorphic, and cb-embedded into $S_{p}$.

The next theorem gives the basic properties of the spaces $Z_{p *}(\sigma)$.

THEOREM 1.5. The space $Z_{p, *}(\sigma)$ has the following properties:

(i) If $\sigma$ satisfies (1.5), then $S_{p}$ is cb-isomorphic to a cb-complemented subspace of $Z_{p, *}(\sigma)$. 
(ii) If $\sigma$ satisfies both (1.5) and (1.6), then $Z_{p, *}(\sigma)$ is not isomorphic to a complemented subspace of $S_{p}$.

Proof. (i): We shall only give the argument for $Z_{p, c_{p}}(\sigma)$. The proof for the other spaces is similar. Let $\left(n_{k}\right) \subseteq \mathbb{N}$ with $\sum_{k=1}^{\infty} \sigma_{n_{k}}^{2 p /(p-2)}<\infty$ and let $V$ consist of those $\left(x, x D_{\sigma}\right) \in Z_{p}(\sigma)$ for which $x_{i j}=0$ unless $j=n_{k}$ for some $k \in \mathbb{N}$. It is readily verified that $V$ is cb-isomorphic to $S_{p}$. From Arazy [1, Theorem 1.1] it follows that $V$ contains another subspace $U$ cb-isomorphic to $S_{p}$ and which is complemented in $Z_{p}(\sigma)$. This shows (i).

(ii): $X_{p}(\sigma)$ can easily be identified with those $\left(x, x D_{\sigma}\right) \in Z_{p}(\sigma)$ for which $x$ is a diagonal matrix. This subspace is clearly contractively complemented in $Z_{p}(\sigma)$. It now follows from Theorem 1.1 that $Z_{p}(\sigma)$ is not isomorphic to a complemented subspace of $S_{p}$.

Before we go on we need the following lemma on non-commutative $L_{p^{-}}$ spaces.

Lemma 1.6. Let $1<p<\infty$ and let $\mathcal{N}$ be a von Neumann algebra so that $L_{p}(\mathcal{N})$ is separable and $L_{p}(0,1)$ does not embed isomorphically into $L_{p}(\mathcal{N})$. Then there exist sequences $\left(I_{k}\right)$ of countable sets and $\left(n_{k}\right) \subseteq \mathbb{N}$ so that

$$
L_{p}(\mathcal{N})=\left(\sum_{k=1}^{\infty} \ell_{p}\left(I_{k}, S_{p}^{n_{k}}\right)\right)_{p} .
$$

Proof. Since $L_{p}(0,1)$ does not embed into $L_{p}(\mathcal{N})$, it follows from a result of Marcolino [21] that $\mathcal{N}$ is a type I factor and therefore the separability of $L_{p}(\mathcal{N})$ and [28] imply that there exist measure spaces $\left(\Omega_{k}, \Sigma_{k}, \mu_{k}\right)$ for all $k \in \mathbb{N}$ and $\left(n_{k}\right) \subseteq \mathbb{N}$ so that

$$
L_{p}(\mathcal{N})=\left(\sum_{n=1}^{\infty} L_{p}\left(\Omega_{k}, \Sigma_{k}, \mu_{k}, S_{p}^{n_{k}}\right)\right)_{p} .
$$

Again, since $L_{p}(0,1)$ does not embed into $L_{p}(\mathcal{N})$, it follows that all the measure spaces on the right side of (1.27) are purely atomic.

We are now able to prove:

THEOREM 1.7. If $\sigma$ satisfies (1.5) and (1.6), then none of the spaces $X_{p}(\sigma), Y_{p}(\sigma)$ or $Z_{p}(\sigma)$ is isomorphic to an $L_{p}(\mathcal{N})$-space where $\mathcal{N}$ is a von Neumann algebra.

Proof. Let $V$ be one of the spaces above and assume that there exists a von Neumann algebra $\mathcal{N}$ so that $V$ is isomorphic to $L_{p}(\mathcal{N})$. Since it follows from [2, Theorem 6] that $L_{p}(0,1)$ does not embed into $S_{p}, L_{p}(\mathcal{N})$ has the form of (1.26) by Lemma 1.6 and therefore it is isomorphic to a complemented subspace of $S_{p}$. This contradicts Theorems 1.1, 1.4, and 1.5 above. 
2. The operator space structure of the classical Rosenthal sequence spaces. In this section we wish to discuss the operator space structure of the Rosenthal sequence spaces defined in Section 1, and it turns out that the local structure of these spaces behaves quite differently. However, due to the non-commutative Burkholder-Rosenthal inequalities [13], [14] the probabilistic viewpoint from the commutative case is still adequate to determine this structure.

Let $\left(\sigma_{i}\right) \subset[0,1]$ and let $A_{i} \subset[0,1], i \in \mathbb{N}$, be intervals of measure $\mu\left(A_{i}\right)=\sigma_{i}^{r}$, where $1 / 2=1 / p+1 / r$. We define $f_{i}\left(\left(t_{j}\right)\right)=\mu\left(A_{i}\right)^{-1 / p} 1_{A_{i}}\left(t_{i}\right)$ for all sequences $\left(t_{j}\right) \subseteq[0,1]$. The sequence $\left(f_{i}\right)_{i \in \mathbb{N}}$ is a sequence of independent random variables on $[0,1]^{\mathbb{N}}$. For sequences $\left(s_{i}\right)$ with finite support we define

$$
\begin{aligned}
u\left(\left(s_{i}\right)\right) & =\sum_{i=1}^{\infty} s_{i} \varepsilon_{i} f_{i}, \\
u_{c}\left(\left(s_{i}\right)\right) & =\sum_{i=1}^{\infty} s_{i} e_{i, 1} \varepsilon_{i} f_{i}, \quad u_{r}\left(\left(s_{i}\right)\right)=\sum_{i=1}^{\infty} s_{i} e_{1, i} \varepsilon_{i} f_{i},
\end{aligned}
$$

where $\left(\varepsilon_{i}\right)$ denotes the sequence of Rademacher functions on $[0,1]$.

Following Rosenthal's argument from [26] using [14] we can now obtain

Proposition 2.1. Let $2 \leq p<\infty$. Then $u, u_{c}$, and $u_{r}$ are cb-isomorphisms between $X_{p}(\sigma), X_{p, c_{p}}(\sigma)$, and $X_{p, r_{p}}(\sigma)$ and their respective images in $L_{p}\left([0,1]^{\mathbb{N}}\right), L_{p}\left([0,1]^{\mathbb{N}} ; C_{p}\right)$, and $L_{p}\left([0,1]^{\mathbb{N}} ; R_{p}\right)$. The images are cb-complemented in the respective spaces.

Proof. We shall only prove the proposition for $u_{c}$ since the other cases go similarly. Let $\left(x_{i}\right)_{i=1}^{n} \subseteq S_{p}$ be arbitrary. From [14, Corollary 1.5] and Proposition 0.2 we get, letting $\sim$ denote two-sided inequalities with constants only depending on $p$,

$$
\begin{aligned}
& \left\|\sum_{i=1}^{n} x_{i} \otimes \varepsilon_{i} f_{i} e_{i 1}\right\|_{S_{p}\left[L_{p}\left((0,1) ; C_{p}\right)\right]} \\
& \sim \max \left\{\left(\sum_{i=1}^{n}\left\|x_{i}\right\|_{S_{p}}^{p}\left\|f_{i}\right\|_{p}^{p}\right)^{1 / p},\left\|\left(\sum_{i=1}^{n} x_{i}^{*} x_{i} \mathbb{E}\left(f_{i}^{2}\right)\right)^{1 / 2}\right\|_{S_{p}},\right. \\
& \left.\left.\sim\left\|\sum_{i=1}^{n}\right\| x_{i} \|_{S_{p}}^{p} \mathbb{E}\left(f_{i}^{2}\right)^{p / 2}\right)^{1 / p}\right\}\left(e_{i i} \oplus \sigma_{i} e_{i 1}\right) \|_{S_{p}\left[X_{p, c_{p}}(\sigma)\right]} .
\end{aligned}
$$

where in the last equivalence we have used the fact that for all $1 \leq i \leq n$ we have $\left\|f_{i}\right\|_{p}=1, \mathbb{E}\left(f_{i}^{2}\right)=\sigma_{i}^{2}$, and $\mathbb{E}\left(f_{i}^{2}\right)^{p / 2}=\mu\left(A_{i}\right)^{p / 2-1} \leq 1$. By Lemma 0.1, $u_{c}$ is a cb-isomorphism. 
For every $1 \leq i \leq n$ we put $f_{i}^{\prime}=\mu\left(A_{i}\right)^{1 / p^{\prime}} 1_{A_{i}}$ and $u_{p^{\prime}}\left(\left(s_{i}\right)\right)=\sum s_{i} \varepsilon_{i} f_{i}^{\prime}$. Using the second part of [14, Theorem 0.1] in much the same way as above we deduce that $u_{p^{\prime}}$ acts as a cb-bounded operator from $X_{p, c_{p}}^{*}$ to $L_{p^{\prime}}(0,1)$. It is readily verified that $u_{c} u_{p^{\prime}}^{*}$ is a cb-projection of $L_{p}(0,1)$ onto the range of $u_{c}$.

Corollary 2.2. The spaces $X_{p}(\sigma), X_{p, c_{p}}(\sigma)$, and $X_{p, r_{p}}$ have the $\gamma_{p}-A P$. More precisely, we have an approximate diagram

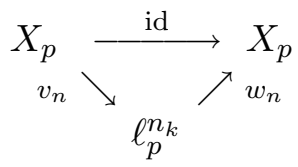

For $X_{p, c_{p}}(\sigma)$ and $X_{p, r_{p}}(\sigma)$ we replace $\ell_{p}^{n_{k}}$ by $\ell_{p}^{n_{k}}\left(C_{p}^{n_{k}}\right)$ and $\ell_{p}^{n_{k}}\left(R_{p}^{n_{k}}\right)$, respectively.

Corollary 2.3. If $\sigma$ satisfies (1.5), then the Rosenthal spaces $X_{p}(\sigma)$ are $\mathcal{C O} \mathcal{L}_{p}$-spaces.

Proof. Follow the proof of [11, Proposition 2.4], using Corollary 2.2 and the fact that $X_{p}(\sigma)$ contains completely complemented copies of $\ell_{p}^{n}$ 's far out.

In the following we want to show that the Rosenthal spaces $X_{p, c_{p}}(\sigma)$ and $X_{p, r_{p}}(\sigma)$ are no longer $\mathcal{O} \mathcal{L}_{p}$. Indeed, the mixture between the Hilbert space structure and the $\ell_{p}$ structure forms the crucial obstacle.

Lemma 2.4. If $1 \leq p<\infty$ and $\mathcal{N}$ is a finite von Neumann algebra, then $C_{p}$ is not cb-isomorphic to a subspace of $R_{p}\left(L_{p}(\mathcal{N})\right)$. Similarly, $R_{p}$ is not cb-isomorphic to a subspace of $C_{p}\left(L_{p}(\mathcal{N})\right)$.

Proof. Suppose to the contrary that $C_{p}$ is isomorphic to a subspace of $R_{p}\left(L_{p}(\mathcal{N})\right)$. Using the natural isomorphism between $R_{p}\left(R_{p}\right)$ and $R_{p}$, we deduce that $S_{p}=R_{p}\left(C_{p}\right)$ is a Banach space isomorphic to a subspace of $R_{p}\left(L_{p}(\mathcal{N})\right) \subset L_{p}\left(B\left(\ell_{2}\right) \otimes \mathcal{N}\right)$. However, for $x \in R_{p}\left(L_{p}(\mathcal{N})\right)$ and $p \geq 2$,

$$
\|x\|_{2}=\left\|x x^{*}\right\|_{L_{1}(\mathcal{N})}^{1 / 2} \leq\left\|x x^{*}\right\|_{p / 2}^{1 / 2} \leq\|x\|_{p} .
$$

So $R_{p}\left(L_{p}(\mathcal{N})\right)$ is isomorphic to a subspace of $L_{p}\left(B\left(\ell_{2}\right) \otimes \mathcal{N}\right) \cap L_{2}\left(B\left(\ell_{2}\right) \otimes \mathcal{N}\right)$ for $2 \leq p<\infty$. For $1 \leq p \leq 2$ a similar argument shows that $R_{p}\left(L_{p}(\mathcal{N})\right)$ is isomorphic to a subspace of $L_{p}\left(\mathcal{N} \otimes B\left(\ell_{2}\right)\right)+L_{2}\left(\mathcal{N} \otimes B\left(\ell_{2}\right)\right)$. According to [9] these spaces are isomorphic to complemented subspaces of $L_{p}(\mathcal{M})$ for some finite von Neumann algebra $\mathcal{M}$. Hence, $S_{p}$ is isomorphic to a subspace of $L_{p}(\mathcal{M})$. This contradicts Sukochev's result for $p \geq 2$, [27], or [6] for $1 \leq p \leq 2$. By symmetry the same holds for $R_{p}$ and $C_{p}$ interchanged.

Corollary 2.5. Let $2<p, r<\infty$ and $1 / 2=1 / p+1 / r$. If $\sigma \notin \ell_{r}$, then the spaces $X_{p, c_{p}}(\sigma)$ and $X_{p, r_{p}}(\sigma)$ are not cb-isomorphic to subspaces of $L_{p}(\mathcal{N})$ with $\mathcal{N}$ finite. 
Proof. Assume first that there is an infinite set $A \subset \mathbb{N}$ so that $\inf _{k \in A} \sigma_{k}$ $>0$. By interpolation we deduce that for the bounded sequence $\left(\sigma_{k}^{-1}\right)_{k \in A}$ the diagonal map $D_{\sigma^{-1}}: C_{p} \rightarrow \ell_{p}$ is completely bounded. Hence, the subspace of $X_{p, c_{p}}(\sigma)$ consisting of the sequences having their support in $A$ is cb-isomorphic to $C_{p}$. In particular, it cannot embed into $L_{p}(\mathcal{N})$ cbisomorphically. Thus $X_{p, c_{p}}(\sigma)$ cannot embed either in this case.

Since $\sum_{j} \sigma_{j}^{r}=\infty$, in the general case we can find disjoint finite subsets $A_{j}$ such that if

$$
\beta_{j}=\left(\sum_{i \in A_{j}} \sigma_{i}^{r}\right)^{1 / r},
$$

then $\inf \beta_{j}>0$. Proposition 1.2 shows that $X_{p, c_{p}}(\beta)$ is cb-isomorphic to a subspace of $X_{p, c_{p}}(\sigma)$ and by the above cb-isomorphic to $C_{p}$, and hence the assertion follows. A similar argument applies for the row spaces.

Lemma 2.6. If $1 \leq p \leq \infty$ and $\mathcal{U}$ is a free ultrafilter on $\mathbb{N}$, then $\prod_{\mathcal{U}} \ell_{p}$ is completely isometrically isomorphic to $L_{p}(\mathcal{N})$ for a commutative von Neumann algebra $\mathcal{N}$.

Proof. Let $\mathcal{N}=\left(\prod_{\mathcal{U}} \ell_{1}\right)^{*}$. From Raynaud's theorem [24] we deduce that for all $n \in \mathbb{N},\left(S_{1}^{n}\left(\prod_{\mathcal{U}} \ell_{1}\right)\right)^{*}=M_{n}(\mathcal{N})$, where $\mathcal{N}$ is a commutative von Neumann algebra obtained as the weak closure of $\prod \ell_{\infty}$. Together with [23, Lemma 5.4] this implies that

$$
L_{p}\left(M_{n} \otimes \mathcal{N}\right)=\prod S_{p}^{n}\left(\ell_{p}\right)=S_{p}^{n}\left(\prod_{\mathcal{U}} \ell_{p}\right)=S_{p}^{n}\left(L_{p}(\mathcal{N})\right) .
$$

Thus $\prod_{\mathcal{U}} L_{p}$ is completely isometrically isomorphic to $\ell_{p}(\mathcal{N})$.

Our aim is now to show that $X_{p, c_{p}}(\sigma)$ is not a rectangular $\mathcal{O} \mathcal{L}_{p}$-space.

Lemma 2.7. If $2 \leq p \leq \infty$, then for all $n \in \mathbb{N}$,

$$
n^{1 / 2-1 / p} \leq \inf _{E \subset C_{p}\left(L_{p}(0,1)\right)} d_{\mathrm{cb}}\left(R_{p}^{n}, E\right) \leq c_{p} n^{1 / 2-1 / p} .
$$

The same estimates hold if $R_{p}$ and $C_{p}$ are interchanged.

Proof. By interpolation,

$$
d_{\mathrm{cb}}\left(R_{p}^{n}, R_{p}^{n} \cap C_{p}^{n}\right) \leq\left\|\mathrm{id}: R_{p}^{n} \rightarrow C_{p}^{n}\right\|_{\mathrm{cb}}\left\|\mathrm{id}: R_{p}^{n} \cap C_{p}^{n} \rightarrow R_{p}^{n}\right\|_{\mathrm{cb}} \leq n^{1 / 2-1 / p} .
$$

By the non-commutative Khinchin inequality [20],

$$
d_{\mathrm{cb}}\left(R_{p}^{n} \cap C_{p}^{n}, \operatorname{span}\left\{g_{j} \mid j=1, \ldots, n\right\}\right) \leq c_{p},
$$

where the $g_{j}$ 's are independent Gaussian variables. To prove the lower estimate, we consider $E \subset L_{p}\left(C_{p}\right)$ and a completely bounded contractive iso- 
morphism $\phi: R_{p}^{n} \rightarrow E$. Let $x_{i}=\phi\left(e_{1 i}\right)$. Then

$$
\begin{aligned}
&\left(\int\left(\sum_{i=1}^{n}\left\|x_{i}(s)\right\|_{2}^{2}\right)^{p / 2} d \mu(s)\right)^{1 / p}=\left\|\sum_{i=1}^{n} e_{i, 1} \otimes x_{i}\right\|_{L_{p}\left(C_{p}^{n}\left(C_{p}\right)\right)} \\
& \leq\|\phi\|_{\mathrm{cb}}\left\|\sum_{i=1}^{n} e_{i, 1} \otimes e_{1, i}\right\|_{C_{p}^{n}\left[R_{p}^{n}\right]}=\|\mathrm{id}\|_{S_{p}^{n}}=n^{1 / p} .
\end{aligned}
$$

However, this implies

$$
\begin{aligned}
\sqrt{n} & =\left(\mathbb{E}\left\|\sum_{i=1}^{n} \varepsilon_{i} e_{1, i}\right\|_{2}^{2}\right)^{1 / 2}=\left(\mathbb{E}\left\|\sum_{i=1}^{n} \varepsilon_{i} \phi^{-1}\left(x_{i}\right)\right\|_{2}^{2}\right)^{1 / 2} \\
& \leq\left\|\phi^{-1}\right\|\left(\mathbb{E}\left\|\sum_{i=1}^{n} \varepsilon_{i}\left(x_{i}\right)\right\|_{L_{p}\left(\ell_{2}\right)}^{2}\right)^{1 / 2} \\
& \leq\left\|\phi^{-1}\right\|\left(\int\left(\mathbb{E}\left\|\sum_{i=1}^{n} \varepsilon_{i} x_{i}(s)\right\|_{2}^{2}\right)^{p / 2} \mu(s)\right)^{1 / p} \\
& =\left\|\phi^{-1}\right\|\left(\int\left(\sum_{i=1}^{n}\left\|x_{i}(s)\right\|_{2}^{2}\right)^{p / 2} \mu(s)\right)^{1 / p} \leq\left\|\phi^{-1}\right\| n^{1 / p}
\end{aligned}
$$

The assertion is proved.

Using a similar idea we can even prove a slighly stronger statement.

Lemma 2.8. If $2 \leq p \leq \infty$, then for all $n \in \mathbb{N}$,

$$
\frac{1}{c_{p}} n^{1 / 2-1 / p} \leq \inf _{E \in Q S\left(\prod_{\mathcal{U}} L_{p}\left(C_{p}\right)\right)} d_{\mathrm{cb}}\left(R_{p}^{n}, E\right) \leq c_{p} n^{1 / 2-1 / p} .
$$

Here $c_{p}$ is an absolute constant and $Q S\left(\prod_{\mathcal{U}} L_{p}\left(C_{p}\right)\right)$ stands for the class of quotients of subspace of ultraproducts of $C_{p}\left(L_{p}(0,1)\right)$. The same estimate holds on exchanging $R_{p}$ with $C_{p}$.

Proof. Let $T: C_{p}^{n} \rightarrow L_{p}(0,1)$ be defined by $T\left(e_{i 1}\right)=\varepsilon_{i}$, where $\left(\varepsilon_{i}\right)_{i=1}^{n}$ are the Bernoulli random variables. We will use a sequence $\left(g_{j}\right)$ of independent normalized complex gaussian random variables on $\left(\Omega^{\prime}, \mu^{\prime}\right)$. Let $h_{1}, \ldots, h_{n} \in$ $L_{p}\left(\Omega, \mu ; \ell_{2}\right)$. Then we deduce from the Khinchin/Kahane's inequality [16] that

$$
\begin{aligned}
& \left\|\sum_{i=1}^{n} \varepsilon_{i} h_{i}\right\|_{L_{p}\left(\ell_{2}\right)} \\
& \quad=\left\|g_{1}\right\|_{p}^{-1}\left(\int_{\Omega \times \Omega^{\prime}} \int_{0}^{1}\left|\sum_{i=1}^{n} \sum_{=1}^{\infty} \varepsilon_{i}(s) g_{j}\left(\omega^{\prime}\right) h_{i}(j, \omega)\right|^{p} d s d \mu^{\prime}\left(\omega^{\prime}\right) d \mu(\omega)\right)^{1 / p}
\end{aligned}
$$




$$
\begin{aligned}
& \leq\left\|g_{1}\right\|_{p}^{-1} c_{0} \sqrt{p}\left(\left.\left.\int_{\Omega \times \Omega^{\prime}}\left(\sum_{i=1}^{n} \mid \sum_{j=1}^{\infty} g_{j}\left(\omega^{\prime}\right) h_{i}(j, \omega)\right)\right|^{2}\right|^{p / 2} d \mu^{\prime}\left(\omega^{\prime}\right) d \mu(\omega)\right)^{1 / p} \\
& \leq\left\|g_{1}\right\|_{p}^{-1} c_{0}^{2} p\left(\int_{\Omega}\left(\sum_{i=1}^{n} \sum_{j=1}^{\infty}\left|h_{i}(j, \omega)\right|^{2}\right)^{p / 2} d \mu(\omega)\right)^{1 / p} .
\end{aligned}
$$

Since for $p \geq 2$, we have $\left\|g_{1}\right\|_{p} \sim \sqrt{p}$, we deduce

$$
\left\|T \otimes \operatorname{id}_{C_{p}\left(L_{p}(\Omega)\right)}: C_{p}^{n}\left(C_{p}\left(L_{p}(\Omega)\right)\right) \rightarrow C_{p}\left(L_{p}([0,1] \times \Omega)\right)\right\| \leq c_{0}^{3} \sqrt{p} .
$$

This remains true if we pass to an ultraproduct and then to a quotient of a subspace. On the other hand, we have seen in Lemma 2.7 that

$$
\left\|T \otimes \operatorname{id}_{R_{p}^{n}}\right\| \geq n^{1 / 2-1 / p} .
$$

Therefore the distance is greater that $n^{1 / 2-1 / p} / c_{0}^{3} \sqrt{p}$.

The next lemma is a kind of "folklore" but for the convenience of the reader we give a proof.

LEMmA 2.9. Let $\mathcal{M}$ be a von Neumann algebra and $2<p \leq \infty, 2 \leq r$ $<\infty$ with $1 / 2=1 / p+1 / r$. Let $F \subset L_{p}(\mathcal{M})$ be a subspace and $T: F \rightarrow R_{p}$ be a linear map. Then $T$ is a complete contraction if and only if there exists a norm one element $a \in L_{r}(\mathcal{M})$ and a contraction $W: L_{2}(\mathcal{M}) \rightarrow \ell_{2}$ such that

$$
T(x)=W(a x) \quad \text { for all } x \in L_{p}(\mathcal{N}) .
$$

In particular, $T$ admits a completely contractive extension $\hat{T}: L_{p}(\mathcal{M})$ $\rightarrow R_{p}$. Similarly, every complete contraction $T: F \rightarrow C_{p}$ has a completely contractive extension of the form $T(x)=W(x a)$.

Proof. Let $\left(x_{j}\right)$ be a finite sequence in $F$. Then

$$
\begin{aligned}
\left(\sum_{j}\left\|T\left(x_{j}\right)\right\|_{2}^{2}\right)^{1 / 2} & =\left\|\sum_{j} e_{j, 1} \otimes T\left(x_{j}\right)\right\|_{R_{p}\left(R_{p}\right)} \leq\left\|\sum_{j} e_{j, 1} \otimes x_{j}\right\|_{R_{p}\left(L_{p}(\mathcal{M})\right)} \\
& =\left\|\sum_{j} x_{j} x_{j}^{*}\right\|_{p / 2}=\sup _{a \geq 0,\|a\|_{r / 2} \leq 1}\left(\sum_{j} \operatorname{tr}\left(a x_{j} x_{j}^{*}\right)\right)^{1 / 2} .
\end{aligned}
$$

Let $B$ be the positive part of the unit ball of $L_{r / 2}(\mathcal{M})$. The function $f_{x}(a) \mapsto$ $\operatorname{tr}\left(a x^{*} x\right)$ is continuous with respect to the weak* topology. Hence, the standard separation yields a probability measure $\mu$ on $B$ such that

$$
\|T(x)\|_{2}^{2} \leq \int_{B} \operatorname{tr}\left(a x^{*} x\right) d \mu(a)=\operatorname{tr}\left(\left(\int_{B} a d \mu(a)\right) x^{*} x\right) .
$$

By convexity, $b=\left(\int_{B} a d \mu(a)\right) \in B$ and therefore

$$
\|T(x)\|_{2} \leq\left\|b^{1 / 2} x\right\|_{2} \text {. }
$$


Let $H=\left\{b^{1 / 2} x \mid x \in F\right\} \subset L_{2}(\mathcal{M})$. Thus there is a linear contraction $W_{1}: H \rightarrow \ell_{2}$ such that $W_{1}\left(b^{1 / 2} x\right)=T(x)$. If $P$ denotes the orthogonal projection onto $H$, then $W=W_{1} P$ satisfies the assertion. To prove the converse, we assume $T(x)=W(a x)$ for some $a \in L_{r}(\mathcal{M})$ of norm less than one. Let $L_{a}: L_{p}(\mathcal{M}) \rightarrow L_{2}(\mathcal{M})^{r_{p}}$ be the left multiplication $L_{a}(x)=a x$. Let $\phi: L_{p / 2} \rightarrow \mathbb{C}$ be the induced linear functional $\phi(y)=\operatorname{tr}\left(y a^{*} a\right)$ of norm less than one. If $x \in L_{p}\left(B\left(\ell_{2}\right) \otimes \mathcal{M}\right)$, we deduce that for every functional the cb-norm coincides with the norm

$$
\begin{gathered}
\left\|\left(\mathrm{id} \otimes L_{a}\right)(x)\right\|_{S_{p}\left(L_{2}(\mathcal{M})^{r_{p}}\right)}=\left\|(\mathrm{id} \otimes \operatorname{tr})\left((a \otimes \mathrm{id}) x x^{*}\left(a^{*} \otimes \mathrm{id}\right)\right)\right\|_{S_{p / 2}}^{1 / 2} \\
=\left\|(\mathrm{id} \otimes \operatorname{tr})\left(x x^{*}\left(a^{*} a \otimes \mathrm{id}\right)\right)\right\|_{S_{p / 2}}^{1 / 2}=\left\|(\mathrm{id} \otimes \phi)\left(x x^{*}\right)\right\|_{S_{p / 2}}^{1 / 2} \\
\leq\left\|x x^{*}\right\|_{S_{p / 2}}^{1 / 2}=\|x\|_{p} .
\end{gathered}
$$

By homogeneity of $L_{2, r_{p}}$, this implies $\left\|W L_{a}\right\|_{\mathrm{cb}} \leq\|W\|\|a\|_{r}$.

Corollary 2.10. If $T: X_{p, c_{p}}(\sigma) \rightarrow C_{p}$ is completely bounded, then $T$ admits a cb-extension to $\ell_{p} \oplus_{p} C_{p}$.

Proposition 2.11. If $2<p<\infty$ and $\mathcal{N}$ is a finite von Neumann algebra, then $\ell_{p}\left(C_{p}\right)$ is not cb-isomorphic to a subspace of $C_{p} \oplus_{p} R_{p}\left(L_{p}(\mathcal{N})\right)$.

Proof. Let $2<r \leq \infty$ be such that $1 / 2=1 / p+1 / r$. Let $T=\left(T^{(1)}, T^{(2)}\right)$ : $\ell_{p}\left(C_{p}\right) \rightarrow C_{p} \oplus_{p} L_{p}(\mathcal{N}) \oplus_{p} R_{p}\left(L_{p}(\mathcal{N})\right)$ be a complete contraction and $T^{-1}$ : $r g(T) \rightarrow \ell_{p}\left(C_{p}\right)$ be a completely bounded inverse with $\left\|T^{-1}\right\|_{\mathrm{cb}} \leq C$. We consider the complete contraction $T_{1}: \ell_{p}\left(S_{p}\right) \rightarrow C_{p}$ defined by $T_{1}(x)=$ $T^{(1)}(P(x)), P$ the projection onto the column space. According to Lemma 2.9, we can find $a \in \ell_{r}\left(S_{r}\right)$ and $W: \ell_{2}\left(S_{2}\right) \rightarrow \ell_{2}$ such that $T_{1}(x)=$ $W(x a)$. Let $\varrho=\left(\|a(i)\|_{r}\right)$ and consider the operator $D_{\varrho}: \ell_{p} \rightarrow \ell_{2}$. We define the bounded map $W^{\prime}: \ell_{2}\left(\ell_{2}\right) \rightarrow \ell_{2}$ by $W^{\prime}\left(\left(x_{i}\right)\right)=W\left(\left(\varrho_{i}^{-1} x_{i} a_{i}\right)\right)$. In particular, we can find an $n$ such that

$$
\left(\sum_{k \geq n} \varrho_{k}^{r}\right)^{1 / r} \leq \frac{1}{2 C} .
$$

In the following, we use the spaces $Y_{n}=\operatorname{span}\left\{\sum_{k} e_{k} \otimes x_{k} \mid k>n, x_{k} \in C_{p}\right\}$ and deduce

$$
\left\|\left.T^{(1)}\right|_{Y_{n}}\right\|_{\mathrm{cb}} \leq\left(\sum_{k \geq n_{1}} \varrho_{k}^{r}\right)^{1 / r}\left\|W^{\prime}: \ell_{2}\left(\ell_{2}\right) \rightarrow \ell_{2}\right\| \leq \frac{1}{2 C} .
$$

If $x \in S_{p}\left(Y_{n}\right)$, we deduce

$$
\begin{aligned}
\frac{1}{C}\|x\|_{S_{p}\left(Y_{n}\right)} & \leq\|(\mathrm{id} \otimes T)(x)\|_{p} \leq\left\|\left(\left.\mathrm{id} \otimes T^{(1)}\right|_{Y_{n}}\right)(x)\right\|_{C_{p}}+\|\left(\mathrm{id} \otimes T^{(2)}(x) \|_{p}\right. \\
& \leq \frac{1}{2 C}\|x\|_{S_{p}\left(\ell_{p}\left(C_{p}\right)\right)}+\left\|\left(\mathrm{id} \otimes T^{(1)}\right)(x)\right\|_{p} .
\end{aligned}
$$


Thus

$$
\frac{1}{2 C}\|x\|_{S_{p}\left(Y_{n}\right)} \leq\left\|\left(\mathrm{id} \otimes T^{(1)}\right)(x)\right\|_{S_{p}\left(R_{p}\left(L_{p}(\mathcal{N})\right)\right)} \leq\|x\|_{S_{p}\left(\ell_{p}\left(C_{p}\right)\right)} .
$$

In particular, $C_{p}$ is cb-isomorphic to a subspace of $R_{p}\left(L_{p}(\mathcal{N})\right)$, which contradicts Lemma 2.4.

For the convenience of the reader we quote the following lemma which is used both in the next proposition and in the next section. The lemma is proved in [9] and [13].

Lemma 2.12. Let $\mathcal{M} \subset \mathcal{N}$ be von Neumann algebras, $\phi$ a faithful normal state on $\mathcal{N}$, and $\mathcal{E}: \mathcal{N} \rightarrow \mathcal{M}$ a faithful conditional expectation such that $\left.\phi\right|_{\mathcal{M}} \circ \mathcal{E}=\phi$. Let $D \in L_{1}(\mathcal{M})$ be the density of $\phi$.

(i) If $1 / r+1 / s=1 / p \geq 1$, then $\mathcal{E}$ induces a contractive map $\mathcal{E}_{p}$ : $L_{p}(\mathcal{N}) \rightarrow L_{p}(\mathcal{M})$ such that

$$
\mathcal{E}_{p}(a x y)=a \mathcal{E}(x) b
$$

for all $a \in L_{r}(\mathcal{M}), b \in L_{s}(\mathcal{M})$ and $x \in \mathcal{N}$.

(ii) Let $1 \leq p, p^{\prime} \leq \infty$ with $1 / p+1 / p^{\prime}=1$ and $L_{p}(\mathcal{N}, \mathcal{E})$ be the completion of $\left\{a D^{1 / p} \mid a \phi\right.$-analytic $\}$ with respect to the norm

$$
\left\|a D^{1 / p}\right\|_{L_{p}(\mathcal{N}, \mathcal{E})}=\left\|D^{1 / p} E\left(a^{*} a\right) D^{1 / p}\right\|_{p / 2}^{1 / 2} .
$$

For $p=\infty$, we take the closure with respect to the strong topology. Then

$$
L_{p}(\mathcal{N}, \mathcal{E})^{*}=L_{p^{\prime}}(\mathcal{N}, \mathcal{E})
$$

and the duality is given by the trace on $\mathcal{M}$.

(iii) Let $1 \leq p^{\prime} \leq 2 \leq p \leq \infty$ with $1 / p+1 / p^{\prime}=1$. Then

$$
\begin{array}{ll}
\|x\|_{L_{p}(\mathcal{N}, \mathcal{E})} \leq\|x\|_{p} \quad \text { for all } x \in L_{p}(\mathcal{M}), \\
\|x\|_{p^{\prime}} \leq\|x\|_{L_{p^{\prime}}(\mathcal{N}, \mathcal{E})} & \text { for all } x \in L_{p^{\prime}}(\mathcal{N}, \mathcal{E}) .
\end{array}
$$

Proposition 2.13. For every separable subspace $W$ of $\prod_{\mathcal{U}} C_{p}\left(L_{p}(0,1)\right)$ there is a commutative von Neumann algebra $\mathcal{N}$ such that $W$ is completely isometrically isomorphic to a subspace of $C_{p}\left(L_{p}(\mathcal{N})\right)$. If in addition $W$ is cbcomplemented, then $W$ can be assumed to be cb-complemented in $C_{p}\left(L_{p}(\mathcal{N})\right)$. The same holds with $R_{p}$ replaced by $C_{p}$.

Proof. Consider the commutative von Neumann algebra $\mathcal{N}=\left(\prod_{\mathcal{U}} L_{1}\right)^{*}$. Let $\iota: \prod_{\mathcal{U}} L_{1} \rightarrow \prod_{\mathcal{U}} L_{1}\left(S_{1}\right)$ be the canonical inclusion map, given coordinatewise by $\iota((f(i)))=\left(e_{00} \otimes f(i)\right)$. Let $q_{0}=\left(e_{00} \otimes 1\right)$ be the projection onto the first corner. Obviously $q \leq q_{0}$ and $\mathcal{E}=\iota^{*}: \prod_{\mathcal{U}} L_{1}\left(S_{1}\right)^{*} \rightarrow \mathcal{N}$ defines a conditional expectation. Let $\mathcal{M}=\prod_{\mathcal{U}} L_{1}\left(S_{1}\right)^{*}$ and consider the norm

$$
\|x\|_{S_{q}^{n}\left(L_{q}(\mathcal{M}, \mathcal{E})\right)}=\left\|(\mathrm{id} \otimes \mathcal{E})\left(x^{*} x\right)^{1 / 2}\right\|_{S_{q}\left(L_{q}(\mathcal{N})\right)}
$$


defined on the space of elements $y d^{1 / q}, d \in L_{1}(\mathcal{N}), y \in L_{q}(N)$. According to Lemma 2.12, we have

$$
L_{p^{\prime}}(\mathcal{M}, \mathcal{E})^{*}=L_{p}(\mathcal{M}, \mathcal{E}) \quad \text { completely isometrically. }
$$

Obviously, the inclusion map $T: \prod_{\mathcal{U}} C_{p^{\prime}}\left(L_{p^{\prime}}(0,1)\right) \rightarrow L_{p^{\prime}}(\mathcal{M}, \mathcal{E})$ is completely isometric and therefore by duality $\prod_{\mathcal{U}} C_{p}\left(L_{p}(0,1)\right)$ is completely contractively complemented in $L_{p}(\mathcal{M}, \mathcal{E})$. Given $x \in S_{p}^{m}(W)$, we see that

$$
\|x\|_{p}^{2}=\left\|x^{*} x\right\|_{p / 2}=\left\|x^{*} x\right\|_{S_{p / 2}^{m}\left[L_{p}(\mathcal{N})\right]} .
$$

Since $\bigcup_{m} S_{p}^{m}[W]$ is separable, we can find a density $D \in L_{1}(\mathcal{N})$ such that

$$
x_{i j}^{*} x_{i j} \leq C(x) D^{1 / p}
$$

for all $x=\left(x_{i j}\right)_{i j=1}^{m}$ in a countable dense subset $\Delta$ of $\bigcup_{m} S_{p}^{m}[W]$. Multiplying with the support projection $q$ of $D$, we can work in $\mathcal{N} q$. For every coordinate $y=x_{i j}, x=\left(x_{i j}\right) \in \Delta$, we consider the polar decomposition

$$
y=u b .
$$

Using Raynaud's isomorphism [24], we see that $b \in L_{p}(q \mathcal{N} q)$. Let $\mathcal{N}_{1}$ be a separable subalgebra generated by the elements $b=b_{i j}(x), x \in \Delta$. Let $\mathcal{M}_{1}$ be a separable subalgebra containing by the polar decompositions $u=u_{i j}(x)$, $x \in \Delta$, such that there exists a conditional expectation $\mathcal{E}_{1}: \operatorname{wcl}\left(\mathcal{M}_{1}\right) \rightarrow \mathcal{N}_{1}$ leaving $\phi$ invariant. Clearly, $W$ is still a (cb-complemented) subspace of $L_{p}\left(\mathcal{M}_{1}, \mathcal{E}\right)$ and we can consider the right $\mathcal{N}_{1}$-module $F$ generated by $M_{1}$ and $\mathcal{N}_{1}$. According to [13], $L_{p}\left(\mathcal{M}_{1}, \mathcal{N}_{1}\right)$ is completely contractively complemented in $C_{p}\left(L_{p}\left(\mathcal{N}_{1}\right)\right)$, and therefore the assertion is proved.

Corollary 2.14. If $2<p<\infty$ and $F$ is a quotient of $R_{p}\left(L_{p}(0,1)\right)$, then $\ell_{p}^{n}\left(C_{p}^{n}\right)$ does not embed uniformly into $C_{p} \oplus_{p} F$.

Proof. Supposing the contrary, we can find $T_{n}=\left(T_{n}^{(1)}, T_{n}^{(2)}\right): \ell_{p}^{n}\left(C_{p}^{n}\right) \rightarrow$ $C_{p} \oplus_{p} F$ such that

$$
\left\|T_{n}\right\|_{\mathrm{cb}} \leq 1 \quad \text { and } \quad\left\|T_{n}^{-1}\right\|_{\mathrm{cb}} \leq C .
$$

Let $\mathcal{U}$ be a free ultrafilter on the natural numbers and define

$$
T: \ell_{p}\left(C_{p}\right) \rightarrow \prod_{\mathcal{U}} C_{p} \oplus_{p} \prod_{\mathcal{U}} F
$$

by $T(x)=\left(\left(T_{n}^{(1)}(x)\right)_{n \in \mathbb{N}},\left(T_{n}^{(2)}(x)\right)_{n \in \mathbb{N}}\right)$. This is well defined because the union $\bigcup_{n} \ell_{p}^{n}\left(C_{p}^{n}\right)$ is norm dense in $\ell_{p}\left(C_{p}\right)$. Moreover, for $x \in S_{p}^{m}\left(\ell_{p}^{n}\left(C_{p}^{n}\right)\right)$, we have

$$
\|(\operatorname{id} \otimes T)(x)\|=\lim _{n^{\prime}>n}\left\|\operatorname{id} \otimes T_{n^{\prime}}(x)\right\|_{S_{p}\left(\ell_{p}\right) \oplus_{p} S_{p}\left(C_{p}\right)} \sim_{C}\|x\|_{S_{p}^{m}\left(\ell_{p}^{n}\left(C_{p}^{n}\right)\right)} .
$$

Denote the first component by $T^{(1)}$ and the second by $T^{(2)}$. We note that $\prod_{\mathcal{U}} F$ is a quotient space of $\prod_{\mathcal{U}} R_{p}\left(L_{p}(0,1)\right)$. Denote the quotient map by $q$. Then we can find a separable subspace $Y \subset \prod_{\mathcal{U}} R_{p}\left(L_{p}(0,1)\right)$ such 
that the image of $T^{(2)}$ is cb-isomorphic to $q(Y)$. According to Proposition 2.13, we can assume that $Y$ is contained in $R_{p}\left(L_{p}(\mathcal{N})\right)$ for some commutative von Neumann algebra $\mathcal{N}$. Moreover, $\prod_{\mathcal{U}} C_{p}$ is a homogeneous Hilbert space which carries the $C_{p}$ structure. Thus every separable subspace is completely isometric to $C_{p}$. Therefore, we can find an embedding of $\ell_{p}\left(C_{p}\right)$ in $C_{p} \oplus_{p} Y / \operatorname{ker}(q)$. Following the argument in Proposition 2.11, we see that for the first component $T^{(1)}$ and every $\varepsilon>0$ there exists an $n$ such that $\left\|\left.T^{(1)}\right|_{\left\{\left(x_{k}\right) \mid x_{1}=\cdots=x_{n}=0\right\}}\right\|_{\mathrm{cb}} \leq \varepsilon$. Thus $C_{p}$ will be cb-isomorphic to a subspace of a quotient of $R_{p}\left(L_{p}(0,1)\right)$. This contradicts Lemma 2.7.

TheOREM 2.15. Let $\sigma$ tend to 0 and be such that for all $n \in \mathbb{N}$ there is a subset $A_{n}$ of cardinality $n$ such that $\sigma_{i}=\alpha_{n}$ for $i \in A_{n}$ and

$$
\lim _{n} n^{1 / r} \alpha_{n}=\infty \text {. }
$$

Then $X_{p, c_{p}}(\sigma)$ does not admit a cb-factorization through $C_{p} \oplus_{p} F$, where $F$ is a quotient of a subspace of $\prod_{\mathcal{U}} R_{p}\left(L_{p}(0,1)\right)$.

Proof. Supposing the contrary we can write id $=T+S$, where $T$ factors through a quotient $F$ of $\prod_{\mathcal{U}} R_{p}\left(L_{p}(0,1)\right)$ and $S$ factors through $C_{p}$. We denote by $Q$ the projection onto the $C_{p}$ coordinate in $X_{p, c_{p}}(\sigma) \subset \ell_{p} \oplus_{p} C_{p}$. Using Lemma 2.10, we can decompose $S=S_{1}+S_{2}$ so that $S_{1}: \ell_{p} \rightarrow X_{p, c_{p}}$ is a completely bounded operator and $S_{2}: C_{p} \rightarrow X_{p, c_{p}}$ is completely bounded. For a fixed index $i \in I$ we consider

$$
\left(e_{i}, \sigma_{i} e_{i}\right)=S\left(e_{i}, \sigma_{i} e_{i}\right)+T\left(e_{i}, \sigma_{i} e_{i}\right)=\sigma_{i} S_{2}\left(0, e_{i}\right)+S_{1}\left(e_{i}, 0\right)+T\left(e_{i}, \sigma_{i} e_{i}\right) .
$$

Thus

$$
1 \leq\left\|S_{1}\left(e_{i}, 0\right)+T\left(e_{i}, \sigma_{i} e_{i}\right)\right\|+\sigma_{i}\left\|S_{2}\right\| .
$$

Hence for $i \geq i_{0}$ we get $\sigma_{i}\left\|S_{2}\right\| \leq 1 / 2$ and therefore

$$
1 / 2 \leq\left\|S_{1}\left(e_{i}, 0\right)+T\left(e_{i}, \sigma_{i} e_{i}\right)\right\| .
$$

Write

$$
S_{1}\left(e_{i}, 0\right)+T\left(e_{i}, \sigma_{i} e_{i}\right)=(y, \sigma y) .
$$

We have the following alternative: If $\|y\|_{p} \leq\|y \sigma\|_{2}$, then

$$
1 / 2 \leq\left(\|y\|_{p}^{p}+\|y \sigma\|_{2}^{p}\right)^{1 / p} \leq 2\|y \sigma\|_{2} .
$$

Hence

$$
1 / 4 \leq\|y \sigma\|_{2} .
$$

If $\|y \sigma\|_{2} \leq\|y\|_{p}$, we get $1 / 4 \leq\|y\|_{p}$ and thus

$$
\sigma_{i}\|y\|_{p} / 4 \leq\|y \sigma\|_{p} \leq\|y \sigma\|_{2} .
$$

In both cases we deduce

$$
\sigma_{i} / 4 \leq\left\|Q S_{1}\left(e_{i}, 0\right)+Q T\left(e_{i}, \sigma_{i} e_{i}\right)\right\|_{2} .
$$


Now we decompose $Q T=T_{1}+T_{2}$, with $T_{1}$ acting on $\ell_{p}$ and $T_{2}$ acting on $C_{p}$ according to Lemma 2.10. Let $n \in \mathbb{N}$ to be determined later and assume that $\sigma_{i}=\alpha_{n}$ is constant on a set $A_{n}$ of cardinality $n$. Recall that

$$
\left(\sum_{i}\left\|Q S_{1}\left(e_{i}\right)\right\|_{2}^{r}\right)^{1 / r} \leq\left\|Q S_{1}\right\| \leq C_{1}
$$

and

$$
\left(\sum_{i}\left\|T_{1}\left(e_{i}\right)\right\|_{2}^{r}\right)^{1 / r} \leq\left\|T_{1}\right\| \leq C_{2} .
$$

Thus we get, for $C_{3}=\left\|T_{2}\right\|$,

$$
\begin{aligned}
\frac{\alpha_{n} n^{1 / r}}{4} \leq & \left(\sum_{i \in A_{n}}\left\|Q S_{1}\left(e_{i}, 0\right)+Q T\left(e_{i}, \sigma_{i} e_{i}\right)\right\|_{2}^{r}\right)^{1 / r} \\
\leq & C_{1}+C_{2}+\left(\sum_{i \in A_{n}}\left\|T_{2}\left(0, \sigma_{i} e_{i}\right)\right\|_{2}^{r}\right)^{1 / r} \\
\leq & C_{1}+C_{2}+\left(\sum_{i \in A_{n},\left\|T_{2}\left(0, e_{i}\right)\right\| \leq 1 / 16}\left\|T_{2}\left(0, \sigma_{i} e_{i}\right)\right\|_{2}^{r}\right)^{1 / r} \\
& +\left(\sum_{i \in A_{n},\left\|T_{2}\left(0, e_{i}\right)\right\|>1 / 16}\left\|T_{2}\left(0, \sigma_{i} e_{i}\right)\right\|_{2}^{r}\right)^{1 / r} \\
\leq & C_{1}+C_{2}+\alpha_{n} \frac{1}{16} n^{1 / r}+\alpha_{n} C_{3} \operatorname{card}\left\{i \in A_{n} \mid\left\|T_{2}\left(0, e_{i}\right)\right\|>1 / 16\right\} .
\end{aligned}
$$

Hence for $n$ so large that $8\left(C_{1}+C_{2}\right) \leq \alpha_{n} n^{1 / r}$ we get

$$
\frac{1}{16 C_{3}} n^{1 / r} \leq \operatorname{card}\left\{i \in A_{n} \mid\left\|T_{2}\left(0, e_{i}\right)\right\|>1 / 16\right\} .
$$

Hence we can find a subset $B_{n}$ of cardinality $n / C_{3}^{r} 16^{r}$ such that for all $i \in B_{n}$ we have

$$
\left\|T_{2}\left(0, e_{i}\right)\right\|_{2}>1 / 16
$$

Now we consider the map $w: \ell_{2}\left(B_{n}\right) \rightarrow \ell_{2}$ defined by $w\left(e_{i}\right)=T_{2}\left(0, e_{i}\right)$. Defining $\delta=C_{3}^{-1} 32^{-2}$ and $n^{\prime}=$ card $B_{n}$ we deduce, for the approximation numbers of $w$,

$$
\begin{aligned}
\frac{1}{16} \sqrt{n^{\prime}} & \leq \pi_{2}(w) \leq\left(\sum_{k=1}^{n^{\prime}} a_{k}(w)^{2}\right)^{1 / 2} \leq \sqrt{\delta} \sqrt{n^{\prime}}\left\|T_{2}\right\|+a_{\delta n^{\prime}}(w) \sqrt{n^{\prime}} \\
& \leq \frac{1}{32} \sqrt{n^{\prime}}+a_{\delta n^{\prime}}(w) \sqrt{n^{\prime}} .
\end{aligned}
$$

Therefore with $\delta^{\prime}=C_{3}^{-r} 16^{-r}$ we obtain

$$
1 / 32 \leq a_{\delta n^{\prime}}(w)=a_{\delta \delta^{\prime} n}(w) .
$$


Let $u: \ell_{2}\left(B_{n}\right) \rightarrow C_{p} \cong \ell_{2}$ be defined by $u\left(e_{i}\right)=Q T\left(e_{i}, \sigma_{i} e_{i}\right)$. In order to obtain a lower estimate for a proportional approximation number of $u$ we observe

$$
\alpha_{n} w\left(e_{i}\right)=T_{2}\left(0, \sigma_{i} e_{i}\right)=Q T\left(e_{i}, \sigma_{i} e_{i}\right)-T_{1}\left(e_{i}, 0\right)=u\left(e_{i}\right)-T_{1}\left(e_{i}, 0\right) .
$$

Since $T_{1}$ is bounded on $\ell_{p}$, the map $T_{1}^{\prime}: \ell_{2} \rightarrow \ell_{2}$ defined by $e_{i} \mapsto T_{1}\left(e_{i}, 0\right)$ factors through the inclusions map id $\operatorname{id}_{2, p}: \ell_{2} \rightarrow \ell_{p}$ :

$$
\alpha_{n} w-u=T_{1} \operatorname{id}_{p, 2},
$$

Let us recall a result of Carl on the Weyl numbers of $\operatorname{id}_{p^{\prime}, 2}$ :

$$
k^{1 / r} x_{k}\left(\mathrm{id}: \ell_{p^{\prime}} \rightarrow \ell_{2}\right) \leq c_{0} .
$$

Therefore we have

$$
\begin{aligned}
\alpha_{n} / 32 & \leq a_{\delta \delta^{\prime} n}\left(\alpha_{n} w\right)=a_{\delta \delta^{\prime} n}\left(u+\alpha_{n} w-u\right) \\
& \leq a_{\left(\delta \delta^{\prime} / 2\right) n}(u)+a_{\left(\delta \delta^{\prime} / 2\right) n}\left(T_{1} \operatorname{id}_{p, 2}\right)=a_{\left(\delta \delta^{\prime} / 2\right) n}(u)+\left(\frac{2 n}{\delta \delta^{\prime}}\right)^{-1 / r} c_{0}\left\|T_{1}\right\| .
\end{aligned}
$$

Hence for $n$ large enough that $n^{1 / r} \alpha_{n} \geq 128 c_{0}\left\|T_{1}\right\| / \delta \delta^{\prime}$ we obtain

$$
\alpha_{n} / 64 \leq a_{\left(\delta \delta^{\prime} / 2\right) n}(u) .
$$

It follows that we can find a linear map $W: \ell_{2} \rightarrow \ell_{2}$ and a $k=\left(\delta \delta^{\prime} / 2\right) n$ dimensional subspace $H \subset \ell_{2}\left(B_{n}\right)$ such that $\|W\| \leq 64 \alpha_{n}^{-1}$ and $W Q T P_{H}$ $=\operatorname{id}_{H}$.

Note that the cb-norm of the identity mapping id : $C_{p} \rightarrow X_{p, c_{p}}$ is completely contractive and thus we obtain

$$
\operatorname{id}_{H}=W Q T \text { id } P_{H} .
$$

According to our assumption $T=w_{1} v_{1}$, where $v_{1}: X_{p, c_{p}}(\sigma) \rightarrow F, w_{1}: F \rightarrow$ $X_{p, c_{p}}(\sigma)$, and $F$ is a quotient of a subspace of $\prod_{\mathcal{U}} R_{p}\left(L_{p}(0,1)\right)$. We deduce from Lemma 2.8 that

$$
\begin{aligned}
\left(\delta \delta^{\prime} / 2\right) n^{1 / r} & =k^{1 / r} \leq c_{p} \inf _{E \in Q S\left(\prod_{\mathcal{U}} R_{p}\left(L_{p}(0,1)\right)\right)} d_{\mathrm{cb}}\left(C_{p}^{k}, E\right) \\
& \leq c_{p}\|W\|_{\mathrm{cb}}\left\|v_{1}\right\|_{\mathrm{cb}}\left\|w_{1}\right\|_{\mathrm{cb}} \leq \alpha_{n}^{-1} c_{p}\left\|v_{1}\right\|_{\mathrm{cb}}\left\|w_{1}\right\|_{\mathrm{cb}} .
\end{aligned}
$$

Using once more $\lim _{n} n^{1 / r} \alpha_{n}=\infty$, we get a contradiction and the assertion is proved.

THEOREM 2.16. If $V \subseteq \ell_{p} \oplus_{p} C_{p} \oplus_{p} R_{p}$ is a rectangular $\mathcal{O} \mathcal{L}_{p}$-space, then there exists an increasing sequence $\left(X_{j}\right)$ of finite-dimensional subspaces of $V$ with dense union and non-negative integers $k_{j}, m_{j}, n_{j}$ and a constant $K$ such that

$$
d_{\mathrm{cb}}\left(X_{j}, \ell_{p}^{k_{j}} \oplus_{p} C_{p}^{n_{j}} \oplus_{p} R_{p}^{m_{j}}\right) \leq K \quad \text { for all } j \in \mathbb{N} .
$$

In particular, $V$ is cb-isomorphic to a cb-complemented subspace of $L_{p}(0,1) \oplus_{p}$ $C_{p} \oplus_{p} R_{p}$. 
If $V \subseteq \ell_{p} \oplus_{p} C_{p}$, the $R_{p}$-terms in (2.2) disappear and $V$ is cb-isomorphic to a cb-complemented subspace of $L_{p}(0,1) \oplus_{p} C_{p}$. Similarly if $V \subseteq \ell_{p} \oplus_{p} R_{p}$.

Proof. Since $V$ is a rectangular $\mathcal{O} \mathcal{L}_{p}$-space there is an increasing sequence $\left(X_{j}\right)$ of finite-dimensional subspace with dense union and numbers $k(j), n_{j}(i)$ and $m_{j}(i)$ and a constant $K_{1}$ so that

$$
d_{\mathrm{cb}}\left(X_{j},\left(\bigoplus_{i=1}^{k(j)} S_{p}^{n_{j}(i), m_{j}(i)}\right)_{p}\right) \leq K_{1} \quad \text { for all } j \in \mathbb{N} .
$$

For every $n \in \mathbb{N}$ we define

$$
h(n)=\sup \left\{m_{j}(i) \mid n_{j}(i) \geq n\right\} .
$$

If $h(n) \geq n$ for all $n \in \mathbb{N}$, clearly $\left(S_{p}^{n}\right)$ embeds cb-uniformly into $V$ and hence $S_{p}$ is isomorphic to a subspace of an ultrapower of $\ell_{p} \oplus_{p} C_{p} \oplus_{p} R_{p}$ which is a Banach lattice of cotype $p$. This contradicts [22, Theorem 2.1]. Hence there is an $n_{0} \in \mathbb{N}$ such that $h\left(n_{0}\right)<n_{0}$. If $n_{j}(i) \leq n_{0}$, then

$$
d_{\mathrm{cb}}\left(S_{p}^{n_{j}(i), m_{j}(i)}, \ell_{p}^{n_{j}(i)}\left(R_{p}^{m_{j}(i)}\right)\right) \leq n_{0}^{1 / r},
$$

and if $n_{j}(i) \geq n_{0}$, then $m_{j}(i)<n_{0}$ and hence

$$
d_{\mathrm{cb}}\left(S_{p}^{n_{j}(i), m_{j}(i)}, \ell_{p}^{m_{j}(i)}\left(C_{P}^{n_{j}(i)}\right)\right) \leq n_{0}^{1 / r} .
$$

We can therefore find a constant $K_{2}$ and numbers $k_{j}^{\prime}, n_{j}^{\prime}(i)$, and $m_{j}^{\prime}(i)$ so that

$$
d_{\mathrm{cb}}\left(X_{j},\left(\bigoplus_{i=1}^{k_{j}^{\prime}} C_{p}^{n_{j}^{\prime}(i)}\right)_{p} \oplus_{p}\left(\bigoplus_{i=1}^{k_{j}^{\prime}} R_{p}^{m_{j}^{\prime}(i)}\right)_{p}\right) \leq K_{2} \quad \text { for all } j \in \mathbb{N} .
$$

For every $n$ and $j$ we put $A_{j}(n)=\left\{i \leq k_{j}^{\prime} \mid n_{j}^{\prime}(i) \geq n\right\}$ and $f(n)=$ $\sup _{j}\left|A_{j}(n)\right|$. If $f(n) \geq n$ for all $n \in \mathbb{N}$, then clearly $\left(\ell_{p}^{n}\left(C_{p}^{n}\right)\right)$ embeds cbuniformly into $V \subseteq \ell_{p} \oplus_{p} C_{p} \oplus_{p} R_{p}$, which contradicts Corollary 2.14. Hence there is an $n_{0}$ so that $\left|A_{j}\left(n_{0}\right)\right|<n_{0}$ for all $j \in \mathbb{N}$. For every $j$ we then get

$$
\begin{array}{r}
d_{\mathrm{cb}}\left(\left(\bigoplus_{i \in A_{j}\left(n_{0}\right)} C_{p}^{n_{j}^{\prime}}\right)_{p}, C_{p}^{\sum_{i \in A_{j}\left(n_{0}\right)} n_{j}^{\prime}(i)}\right) \leq n_{0}^{1 / r}, \\
d_{\mathrm{cb}}\left(\bigoplus_{i \notin A_{j}\left(n_{0}\right)} C_{P}^{n_{j}^{\prime}(i)}, \ell_{p}^{\sum_{i \notin A_{j}\left(n_{0}\right)} n_{j}^{\prime}(i)}\right) \leq n_{0}^{1 / r} .
\end{array}
$$

Treating the $R_{p}$-terms in the same way we find that there is a constant $K$ and numbers $k_{j}, n_{j}$, and $m_{j}$ so that

$$
d_{\mathrm{cb}}\left(X_{j}, \ell_{p}^{k_{j}} \oplus_{p} C_{p}^{n_{j}} \oplus_{p} R_{p}^{m_{j}}\right) \leq K \quad \text { for all } j \in \mathbb{N},
$$

which proves (2.2). Using an ultraproduct construction as in [5, Section 10.3] we deduce that there is an ultrafilter $\mathcal{U}$ so that $V$ is cb-complemented in $\prod_{\mathcal{U}} \ell_{p} \oplus_{p} \prod_{\mathcal{U}} C_{p} \oplus_{p} \prod_{\mathcal{U}} R_{p}$. Since $\prod_{\mathcal{U}} \ell_{p}$ is cb-isometrically isomorphic to $L_{p}(\mathcal{N})$ for some commutative $\mathcal{N}$, and $C_{p}$ and $R_{p}$ are homogeneous, the separability of $V$ implies that it is cb-complemented in $L_{p}\left(\mathcal{N}_{1}\right) \oplus_{p} C_{p} \oplus_{p} R_{p}$ 
with $\left(\mathcal{N}_{1}\right)_{*}$ separable. Decomposing $\mathcal{N}_{1}$ into discrete and continuous parts we find that $L_{p}\left(\mathcal{N}_{1}\right)$ is cb-contractively complemented in $L_{p}(0,1)$ and hence $V$ is cb-isomorphic to a cb-complemented subspace of $L_{p}(0,1) \oplus_{p} C_{p} \oplus_{p} R_{p}$.

Since $\left(R_{p}^{n}\right)$ does not embed cb-uniformly into $\ell_{p} \oplus_{p} C_{p}$ by Lemma 2.7, it is readily seen that if $V \subseteq \ell_{p} \oplus_{p} C_{p}$, then the $R_{p}$-components disappear in the argument above, and the ultraproduct construction shows that $V$ is cb-isomorphic to a cb-complemented subspace of $L_{p}(0,1) \oplus_{p} C_{p}$.

As a corollary we obtain

Theorem 2.17. If $\sigma$ satisfies (1.5) and (1.6), then the spaces $X_{p, c_{p}}(\sigma)$ and $X_{p, r_{p}}(\sigma)$ are not rectangular $\mathcal{O} \mathcal{L}_{p}$-spaces.

Proof. Suppose that $X_{p, c_{p}}(\sigma)$ is a rectangular $\mathcal{O} \mathcal{L}_{p}$-space. Theorem 2.16 then shows that it is cb-complemented in $L_{p}(0,1) \oplus_{p} C_{p}$. By Theorem 1.3 we can assume that $\sigma$ satisfies the additional assumptions in Theorem 2.15, and hence that theorem yields a contradiction.

THeOREM 2.18. If $\sigma$ satisfies (1.5) and (1.6) and

$$
V \in\left\{R_{p} \oplus_{p} X_{p, c_{p}}(\sigma), \ell_{p}\left(R_{p}\right) \oplus_{p} X_{p, c_{p}}(\sigma), X_{p, r_{p}}(\sigma) \oplus_{p} X_{p, c_{p}}(\sigma)\right\},
$$

then $V$ is not a rectangular $\mathcal{O} \mathcal{L}_{p}$-space.

Proof. Suppose $V=\ell_{p}\left(R_{p}\right) \oplus_{p} X_{p, c_{p}}(\sigma)$. The proof of Theorem 2.16 shows that $V$ is cb-complemented in $C_{p} \oplus_{p} \prod_{\mathcal{U}} R_{p}\left(\ell_{p}\right)$, which contradicts Theorem 2.15 since $X_{p, c_{p}}(\sigma)$ is cb-complemented in $V$. The other cases follow directly from Theorem 2.16.

Proposition 2.19. Assume that $\sigma$ satisfies (1.5) and (1.6), and let $\mathcal{U}$ be a free ultrafilter on the integers.

(i) If $V \in\left\{X_{p, c_{p}}(\sigma), R_{p} \oplus_{p} X_{p, c_{p}}(\sigma), X_{p, r_{p}}(\sigma) \oplus_{p} X_{p, c_{p}}(\sigma)\right\}$, then $\ell_{p}\left(R_{p}\right) \oplus_{p}$ $X_{p, c_{p}}(\sigma)$ does not embed into $\prod_{\mathcal{U}} V$.

(ii) $X_{p, r_{p}}(\sigma) \oplus_{p} X_{p, c_{p}}(\sigma)$ is not cb-isomorphic to a cb-complemented subspace of $\prod_{\mathcal{U}}\left(R_{p} \oplus_{p} X_{p, c_{p}}(\sigma)\right)$.

In particular, the spaces $X_{p, c_{p}}(\sigma), R_{p} \oplus_{p} X_{p, c_{p}}(\sigma), X_{p, r_{p}}(\sigma) \oplus_{p} X_{p, c_{p}}(\sigma)$, $\ell_{p}\left(R_{p}\right) \oplus_{p} X_{p, c_{p}}(\sigma)$ are mutually not cb-isomorphic.

Proof. To prove (i), we observe that $V \subset \ell_{p} \oplus_{p} C_{p} \oplus_{p} R_{p}$. Thus the assertion follows from the row version of Corollary 2.14. To get (ii) we note that $R_{p} \oplus_{p} X_{p, c_{p}}(\sigma)$ is complemented in $R_{p} \oplus_{p} L_{p}\left([0,1] ; C_{p}\right)$. According to Proposition 2.13 a separable complemented subspace of $\prod_{\mathcal{U}} R_{p} \oplus_{p} L_{p}\left([0,1] ; C_{p}\right)$ is cb-complemented in $R_{p} \oplus_{p} C_{p}\left(L_{p}(\mathcal{N})\right)$ for a commutative $\mathcal{N}$. But the row version of Theorem 2.15 excludes this for $X_{p, r_{p}}(\sigma)$.

REMARK 2.20. If $W \in\left\{\ell_{p}\left(R_{p}\right), \ell_{p}\left(R_{p}\right) \oplus_{p} X_{p, c_{p}}(\sigma), R_{p} \oplus_{p} X_{p, c_{p}}(\sigma)\right.$, $\left.X_{p, r_{p}}(\sigma) \oplus_{p} X_{p, c_{p}}(\sigma)\right\}$, then $W$ contains $R_{p}$ cb-isomorphically which does 
not cb-embed into an ultrapower of $L_{p}\left([0,1] ; C_{p}\right)$. However, $X_{p, c_{p}}(\sigma) \subseteq$ $L_{p}\left([0,1] ; C_{p}\right)$ and hence $W$ does not cb-embed into an ultrapower of $X_{p, c_{p}}(\sigma)$.

Consequently, none of the spaces above nor those from Proposition 2.19 can be paved with local pieces of any of the others except for trivial reasons. It is easily seen that we can also add $\ell_{p}\left(C_{p}\right) \oplus_{p} X_{p, r_{p}}(\sigma)$ and the rectangular $\mathcal{O} \mathcal{L}_{p}$-space $\ell_{p}\left(C_{p}\right) \oplus_{p} X_{p, c_{p}}(\sigma)$ to this list.

At the end of this section we want to compare the space $X_{p, c_{p}}(\sigma), X_{p, r_{p}}(\varrho)$ with their intersection in interpolation sense. Let $2<p<\infty$ and let $\sigma=\left(\sigma_{n}\right)$ and $\varrho=\left(\varrho_{n}\right)$ be two positive sequences. In analogy with the spaces defined in Section 1 we let $X(\sigma, \varrho)$ be the subspace of $S_{p} \oplus_{p} C_{p} \oplus_{p} R_{p}$ defined as the closed linear span of the sequence $\left\{e_{n n} \oplus_{p} \sigma_{n} e_{n 1} \oplus_{p} \varrho_{n} e_{1 n}\right\}$. Note that $X(\sigma, \varrho)$ is the interpolation space $X_{p, c_{p}}(\sigma) \cap X_{p, r_{p}}(\varrho)$. We shall show that if $\sigma$ and $\varrho$ satisfy (1.5) and (1.6), then $X_{p}(\sigma, \varrho)$ is a rectangular $\mathcal{O} \mathcal{L}_{p}$-space if and only if it is cb-isomorphic to $X_{p}(\sigma), X_{p}(\sigma) \oplus_{p} C_{p}, X_{p}(\sigma) \oplus_{p} R_{p}$ or $X_{p}(\sigma) \oplus_{p} C_{p} \oplus_{p} R_{p}$. We first investigate the space $X_{p}(\alpha, \beta)$ where $\alpha>0$ and $\beta>0$ are constants. We have:

Proposition 2.21. There is a constant $K=K(p)$ such that if $T$ is a cb-isomorphism of $X_{p}(\alpha, \beta)$ into $L_{p}(0,1) \oplus_{p} C_{p} \oplus_{p} R_{p}$ and $P$ is a cb-projection of $L_{p}(0,1) \oplus_{p} C_{p} \oplus_{p} R_{p}$ onto $T\left(X_{p}(\alpha, \beta)\right)$, then either

$$
\max (\alpha, \beta) \leq K\|T\|_{\mathrm{cb}}\left\|T^{-1}\right\|_{\mathrm{cb}} \min (\alpha, \beta)
$$

or

$$
\frac{1}{2 \min (\alpha, \beta)} \leq K\|P\|_{\mathrm{cb}}\|T\|_{\mathrm{cb}}\left\|T^{-1}\right\|_{\mathrm{cb}} .
$$

If $T$ is a cb-isomorphism of $X_{p, c_{p}}(\alpha)$ into $L_{p}(0,1) \oplus_{p} C_{p}$ and $P$ is a cbprojection of $L_{p}(0,1) \oplus_{p} C_{p}$ onto $T\left(X_{p, c_{p}}(\alpha)\right)$, then

$$
\frac{1}{2 \alpha} \leq K\|P\|_{\mathrm{cb}}\|T\|_{\mathrm{cb}}\left\|T^{-1}\right\|_{\mathrm{cb}} .
$$

Similarly for $X_{p, r_{p}}(\sigma)$.

Proof. Assume that $\beta \leq \alpha$ (the other case can be proved similarly), let $Q_{1}$ be the natural projection of $L_{p}(0,1) \oplus_{p} C_{p} \oplus_{p} R_{p}$ onto $L_{p}(0,1)$, and $Q_{2}$ the natural projection of $L_{p}(0,1) \oplus_{p} C_{p} \oplus_{p} R_{p}$ onto $C_{p} \oplus_{p} R_{p}$. If $\left(f_{n}\right)$ denotes the canonical basis of $X_{p}(\alpha, \beta)$, we put $h_{n}=Q_{1} T f_{n}$ for all $n \in \mathbb{N}$. Since $f_{n} \rightarrow 0$ weakly, so does $\left(h_{n}\right)$ and we can therefore extract a martingale subsequence of $\left(h_{n}\right)$ and then use the argument in [14] to extract a further subsequence, still called $\left(h_{n}\right)$, so that there exist constants $K_{1}=K_{1}(p) \geq 1, b_{1} \geq 0$ and $b_{2} \geq 0$ such that

$$
\begin{aligned}
& \left\|\sum_{k} a_{k} h_{k}\right\|_{S_{p}\left[L_{p}(0,1)\right]} \\
& \quad \sim_{K_{1}} \max \left\{b_{1} \sum_{k}\left\|a_{k}\right\|_{p}^{p}, b_{2}\left\|\left(\sum_{k} a_{k}^{*} a_{k}\right)^{1 / 2}\right\|_{S_{p}}, b_{2}\left\|\left(\sum_{k} a_{k} a_{k}^{*}\right)^{1 / 2}\right\|_{S_{p}}\right\}
\end{aligned}
$$


for all finite sequences $\left(a_{k}\right) \subseteq S_{p}$. Plugging in the vectors $a_{k}=e_{1 k}$, we get, for every $n \in \mathbb{N}$,

$$
\max \left(b_{1} n^{1 / p}, b_{2} n^{1 / p}, b_{2} n^{1 / 2}\right) \leq K_{1}\|T\|_{\mathrm{cb}} \max \left(n^{1 / p}, \alpha n^{1 / p}, \beta n^{1 / 2}\right),
$$

which implies that $b_{2} \leq K_{1} \beta$.

As in Corollary 2.10, there is a constant $K_{2}$ only depending on $p$ such that the operator $Q_{2} T$ has a cb-extension $S: S_{p} \oplus_{p} C_{p} \oplus_{p} R_{p} \rightarrow C_{p} \oplus_{p} R_{p}$ with $\|S\|_{\mathrm{cb}} \leq K_{2}\|T\|_{\mathrm{cb}}$. Hence for all $n \in \mathbb{N}$,

$$
T f_{n}=h_{n}+S e_{n n}+\alpha S e_{n 1}+\beta S e_{1 n} .
$$

By [26], $\sum_{n=1}^{\infty}\left\|S e_{n n}\right\|^{r}<\infty$, and if $Q$ denotes the canonical projection of $S_{p} \oplus_{p} C_{p} \oplus_{p} R_{p}$ onto $R_{p}$ we find that the operator $Q T^{-1} P S \mid C_{p}$ is $(r, 2)$ summing and therefore also $\sum_{n=1}^{\infty}\left\|Q T^{-1} P S e_{n 1}\right\|^{r}<\infty$. In particular, we can find an $n_{0} \in \mathbb{N}$ such that

$$
\left\|T^{-1} P S e_{n n}\right\|+\frac{\alpha}{\beta}\left\|Q T^{-1} P S e_{n 1}\right\| \leq \frac{1}{4} \quad \text { for all } n \geq n_{0} .
$$

If $\left(F_{n}\right)$ denotes the biorthogonal system to $\left(f_{n}\right)$, then clearly $\left|F_{n}\left(T^{-1} P S e_{n 1}\right)\right|$ $\leq(1 / \beta)\left\|Q T^{-1} P S e_{n 1}\right\|$ and hence $(2.6)$ gives

$$
\begin{aligned}
1 & \leq\left|F_{n}\left(T^{-1} P h_{n}\right)\right|+\beta\left|F_{n}\left(T^{-1} P S e_{1 n}\right)\right|+1 / 4 \\
& \leq\left|F_{n}\left(T^{-1} P h_{n}\right)\right|+K_{2} \beta\|P\|_{\mathrm{cb}}\|\| T\left\|_{\mathrm{cb}}\right\| T^{-1} \|_{\mathrm{cb}}+1 / 4
\end{aligned}
$$

for all $n \geq n_{0}$. If we now assume that $K_{2} \beta\|T\|_{\mathrm{cb}}\left\|T^{-1}\right\|_{\mathrm{cb}}\|P\|_{\mathrm{cb}}<1 / 2$, then by the above $1 / 4 \leq\left|F_{n}\left(T^{-1} P h_{n}\right)\right|$ for all $n \geq n_{0}$.

By interpolation there exists a constant $K_{3}=K_{3}(p)$ such that if $U$ denotes the diagonal of $T^{-1} P \mid\left[h_{n}\right]$ with respect to the bases $\left(f_{n}\right)$ and $\left(h_{n}\right)$, then $U$ is cb-bounded with $\|U\|_{\mathrm{cb}} \leq K_{3}\left\|T^{-1}\right\|_{\mathrm{cb}}\|P\|_{\mathrm{cb}}$, and hence for all $\left(a_{k}\right) \subseteq S_{p}$ and all $n \geq n_{0}$ we get

$$
\begin{aligned}
\frac{1}{4}\left\|\sum_{k=n_{0}}^{n} a_{k} \otimes f_{k}\right\| & \leq\left\|U\left(\sum_{k=n_{0}}^{n} a_{k} \otimes h_{k}\right)\right\|_{S_{p}\left[L_{p}(0,1)\right]} \\
& \leq K_{3}\left\|T^{-1}\right\|_{\mathrm{cb}} P\left\|_{\mathrm{cb}}\right\| \sum_{k=n_{0}}^{n} a_{k} \otimes h_{k} \|_{S_{p}\left[L_{p}(0,1)\right]} .
\end{aligned}
$$

If we plug in the vectors $a_{k}=e_{k 1}$ in this inequality we get $\frac{1}{4} \max \left\{\left(n-n_{0}\right)^{1 / p}, \alpha\left(n-n_{0}\right)^{1 / 2}, \beta\left(n-n_{0}\right)^{1 / p}\right\}$ $\leq K_{1} K_{3}\|T\|_{\mathrm{cb}}\left\|T^{-1}\right\|_{\mathrm{cb}}\|P\|_{\mathrm{cb}} \max \left\{b_{1}\left(n-n_{0}\right)^{1 / p}, b_{2}\left(n-n_{0}\right)^{1 / 2}, b_{2}\left(n-n_{0}\right)^{1 / p}\right\}$ and therefore $\alpha \leq K_{1} K_{3}\|T\|_{\mathrm{cb}}\left\|T^{-1}\right\|_{\mathrm{cb}}\|P\|_{\mathrm{cb}} b_{2} \leq K_{1}^{2} K_{3}\|T\| T^{-1}\left\|_{\mathrm{cb}}\right\| P \|_{\mathrm{cb}} \beta$. Hence we have proved the proposition with $K=\max \left(K_{1}^{2} K_{3}, K_{2}\right)$.

To prove the statement for $X_{p, c_{p}}(\alpha)$ we go through the argument above, but we omit the $R_{p}$-coordinate, and adjust the sequence $\left(h_{n}\right)$ to the new 
situation. Then we drop the argument with the projection $Q$. The first part will then show that $b_{2} \leq K_{1} \alpha$. If $K_{2} \alpha\|T\|_{\mathrm{cb}}\left\|T^{-1}\right\|_{\mathrm{cb}}\|P\|_{\mathrm{cb}}<1 / 2$, then the second part will show that $\alpha \leq K_{1} K_{3}\|T\|_{\mathrm{cb}}\left\|T^{-1}\right\|_{\mathrm{cb}}\|P\|_{\mathrm{cb}} b_{2}$. Hence $\left(f_{n}\right)$ is cb-equivalent to $\left(h_{n}\right)$, which is a contradiction because $X_{p, c_{p}}(\alpha)$ is cbisomorphic to $C_{p}$, which does not embed into $L_{p}(0,1)$ by Lemma 2.4.

We need the following two lemmas:

Lemma 2.22. Let $2 \leq p<\infty$ and let $\sigma$ and $\varrho$ be two sequences such that there exist $\delta>0$ and $\varepsilon>0$ with $\sigma_{n} \leq \delta \varrho_{n}$ for all $n \in \mathbb{N}$ and $\sum_{\sigma_{n} \leq \varepsilon} \sigma_{n}^{r}<\infty$. If $X_{p}(\sigma, \varrho)$ is cb-isomorphic to a cb-complemented subspace of $L_{p}(0,1) \oplus_{p}$ $C_{p} \oplus_{p} R_{p}$, then there exist $0 \leq K, M, N \leq \infty$ such that $X_{p}(\sigma, \varrho)$ is cbisomorphic to $l_{p}^{N} \oplus_{p}\left(C_{p} \cap R_{p}\right)^{M} \oplus_{p} R_{p}^{K}$. If $\varrho_{n} \rightarrow 0$, the last two summands do not occur.

Proof. Assume that $X_{p}(\sigma, \varrho)$ is an $\mathcal{O} \mathcal{L}_{p^{-}}$-space, put

$$
A=\left\{n \in \mathbb{N} \mid \sigma_{n} \leq \varepsilon\right\}, \quad B=\left\{n \in \mathbb{N} \mid \sigma_{n}>\varepsilon\right\},
$$

and let $\sigma_{A}=\left\{\sigma_{n} \mid n \in A\right\}$ and $\sigma_{B}=\left\{\sigma_{n} \mid n \in B\right\}$. In a similar manner we define $\varrho_{A}$ and $\varrho_{B}$. Clearly we can write

$$
X_{p}(\sigma, \varrho)=X_{p}\left(\sigma_{A}, \varrho_{A}\right) \oplus X_{p}\left(\sigma_{B}, \varrho_{B}\right) .
$$

If $\liminf \varrho_{A}(n)>0$, then $X_{p}\left(\sigma_{A}, \varrho_{A}\right)$ is cb-isomorphic to $R_{p}^{|A|}$ (which is cb-isomorphic to $\ell_{p}^{|A|}$ in case $A$ is finite). Assume next that $\lim \inf \varrho_{A}(n)=0$. If $\varrho_{A}$ satisfies (1.6), then $X_{p}\left(\sigma_{A}, \varrho_{A}\right)$ is cb-isomorphic to $X_{p, r_{p}}\left(\varrho_{A}\right)$, which contradicts Theorem 2.17, and hence there is an $\varepsilon_{1}>0$ such that $\sum_{\varrho_{A}(n) \leq \varepsilon_{1}} \varrho_{A}(n)^{r}<\infty$. We may assume that $\varepsilon_{1}=\varepsilon$ and conclude that $X_{p}(\sigma, \varrho)$ is cb-isomorphic to $\ell_{p}^{|A|}$. If $n \in B$, then $\varepsilon<\sigma_{n} \leq \delta \varrho_{n}$ so that $X_{p}\left(\sigma_{B}, \varrho_{B}\right)$ is cb-isomorphic to $\left(C_{p} \cap R_{p}\right)^{|B|}$.

Summing up we have found that there exist $0 \leq K, M, N \leq \infty$ such that $X_{p}(\sigma, \varrho)$ is cb-isomorphic to $\ell_{p}^{N} \oplus_{p}\left(C_{p} \cap R_{p}\right)^{M} \oplus_{p} R_{p}^{K}$.

Lemma 2.23. Let $2<p<\infty$ and let $\sigma$ and $\varrho$ be two sequences such that $X_{p}(\sigma, \varrho)$ is cb-complemented in $L_{p}(0,1) \oplus_{p} C_{p} \oplus_{p} R_{p}$. Then $\left\{\varrho_{n} \mid \sigma_{n} \geq \varepsilon\right\}$ does not satisfy (1.6) for any $\varepsilon>0$. The same holds with $\sigma$ and $\varrho$ interchanged.

Proof. Suppose that there is an $\varepsilon>0$ such that $\left\{\varrho_{n} \mid \sigma \geq \varepsilon\right\}$ satisfies (1.6). Then it also satisfies (1.5), and if $\beta>0$ is arbitrary, we can find a sequence $\left(B_{k}\right)$ of mutually disjoint finite subsets of $\mathbb{N}$ so that

$$
\beta \leq\left(\sum_{n \in B_{k}, \sigma_{n} \geq \varepsilon} \varrho_{n}^{r}\right)^{1 / r} \leq 2 \beta .
$$

For every $k \in \mathbb{N}$ we put $\alpha_{k}=\left(\sum_{n \in B_{k}, \sigma_{n} \geq \varepsilon} \sigma_{n}^{r}\right)^{1 / r}$ and arguing as in Proposition 1.2 we find that $X_{p}\left(\left(\alpha_{k}\right), \beta\right)$ is cb-complemented in $X_{p}(\sigma, \varrho)$. Clearly 
$\alpha=\liminf \alpha_{k} \geq \varepsilon$, and if we choose a subsequence $\left(\alpha_{k_{m}}\right)$ tending sufficiently fast to $\alpha$ we conclude that $X_{p}(\alpha, \beta)$ is cb-complemented in $X_{p}(\sigma, \varrho)$, and hence also in $L_{p}(0,1) \oplus_{p} C_{p} \oplus_{p} R_{p}$. This contradicts (2.3) and (2.4) for $\beta$ small enough.

We are now able to prove:

THeOrem 2.24. Let $\sigma$ and $\varrho$ be two sequences satisfying (1.5) and (1.6). If $X_{p}(\sigma, \varrho)$ is a rectangular $\mathcal{O} \mathcal{L}_{p}$-space, then it is cb-isomorphic to $X_{p}(\sigma)$, $X_{p}(\sigma) \oplus_{p} R_{p}, X_{p}(\sigma) \oplus_{p} C_{p}$ or $X_{p}(\sigma) \oplus_{p} C_{p} \oplus_{p} R_{p}$. If in addition $\sigma_{n} \rightarrow 0$ and $\varrho_{n} \rightarrow 0$, then $X_{p}(\sigma, \varrho)$ is cb-isomorphic to $X_{p}(\sigma)$.

Proof. If $X_{p}(\sigma, \varrho)$ is a rectangular $\mathcal{O} \mathcal{L}_{p^{-}}$space, Theorem 2.17 shows that it is cb-isomorphic to a cb-complemented subspace of $L_{p}(0,1) \oplus_{p} C_{p} \oplus_{p}$ $R_{p}$. Suppose that for all $\varepsilon>0$ and all $\delta>0$ we have $\sum_{\left\{\varrho_{n} \leq \delta \sigma_{n}, \varrho_{n} \leq \varepsilon\right\}} \varrho_{n}^{r}$ $=\infty$. We shall show that this leads to a contradiction. Let $\delta>0$ and $A=\left\{n \in \mathbb{N} \mid \varrho_{n} \leq \delta \sigma_{n}\right\}$, and define $\sigma_{A}$ and $\varrho_{A}$ as in Lemma 2.22. Clearly $\varrho_{A}$ satisfies (1.5) and (1.6). If $\sum_{\left\{\sigma_{A}(n) \leq \varepsilon\right\}} \sigma_{A}(n)^{r}<\infty$ for some $\varepsilon>0$, then also $\sum_{\left\{\sigma_{A}(n) \leq \varepsilon\right\}} \varrho_{A}(n)^{r}<\infty$ and therefore $\left\{\varrho_{A}(n) \mid \sigma_{A}(n)>\varepsilon\right\}$ satisfies (1.6), which contradicts Lemma 2.23. Hence also $\sigma_{A}$ satisfies (1.5) and (1.6). Let now $\alpha>0$, choose mutually disjoint finite sets $A_{k} \subseteq \mathbb{N}$ such that for all $k \in \mathbb{N}$,

$$
\alpha \leq\left(\sum_{n \in A_{k}} \sigma_{A}(n)^{r}\right)^{1 / r} \leq 2 \alpha
$$

and put $\beta_{k}=\left(\sum_{n \in A_{k}} \varrho_{A}(n)^{r}\right)^{1 / r}$ for all $k \in \mathbb{N}$. Again Proposition 1.2 shows that $X_{p}\left(\alpha,\left(\beta_{k}\right)\right)$ is cb-isomorphic to a cb-complemented subspace of $X_{p}(\sigma, \varrho)$, and by choosing a subsequence of $\left(\beta_{k}\right)$ tending sufficiently fast to $\beta=\liminf \beta_{k}>0$ we find that $X_{p}(\alpha, \beta)$ is cb-isomorphic to a cbcomplemented subspace of $X_{p}(\sigma, \varrho)$. If $\beta=0$ we have $X_{p}(\alpha, \beta)=X_{p, c_{p}}(\alpha)$, contrary to (2.5) of Proposition 2.21 for $\alpha$ small enough. If $\beta>0$, then $\beta \leq 2 \delta \alpha$, contrary to (2.3) of Proposition 2.21 for $\delta$ small enough. By choosing $\alpha$ small enough, (2.4) is violated and we have reached a contradiction.

Interchanging the roles of $\sigma$ and $\varrho$ in the argument above we can conclude that there are $\varepsilon>0$ and $\delta>0$ such that

$$
\begin{gathered}
\sum_{\left\{\sigma_{n} \leq \delta \varrho_{n}, \sigma_{n} \leq \varepsilon\right\}} \sigma_{n}^{r}<\infty, \\
\sum_{\left\{\varrho_{n} \leq \delta \sigma_{n}, \varrho_{n} \leq \varepsilon\right\}} \varrho_{n}^{r}<\infty .
\end{gathered}
$$

Let $A$ be as above and put

$$
B=\left\{n \in \mathbb{N} \mid \delta \varrho_{n}<\sigma_{n}<(1 / \delta) \varrho_{n}\right\}, \quad D=\left\{n \in \mathbb{N} \mid \sigma_{n} \leq \delta \varrho_{n}\right\},
$$


and define the sequences $\left(\sigma_{A}\right),\left(\sigma_{B}\right),\left(\sigma_{D}\right),\left(\varrho_{A}\right),\left(\varrho_{B}\right)$, and $\left(\varrho_{D}\right)$ as before. We can then write

$$
X_{p}(\sigma, \varrho)=X_{p}\left(\sigma_{A}, \varrho_{A}\right) \oplus X_{p}\left(\sigma_{B}, \varrho_{B}\right) \oplus X_{p}\left(\sigma_{D}, \varrho_{D}\right) .
$$

By Lemma 2.22, $X_{p}\left(\sigma_{A}, \varrho_{A}\right) \oplus X_{p}\left(\sigma_{D}, \varrho_{D}\right)$ is cb-isomorphic to $l_{p}^{N} \oplus_{p}\left(C_{p} \cap\right.$ $\left.R_{p}\right)^{M} \oplus_{p} C_{p}^{K} \oplus_{p} R_{p}^{L}$ for some $0 \leq k, L, M, N \leq \infty$. $X_{p}\left(\sigma_{B}, \varrho_{B}\right)$ is cbisomorphic to $X_{p}\left(\sigma_{B}\right)$ and since $\sigma_{B}$ satisfies (1.5) and (1.6) it contains cb-complemented copies of $l_{p} \oplus_{p}\left(C_{p} \cap R_{p}\right)$, which shows that $X_{p}(\sigma, \varrho)$ is cb-isomorphic to $X_{p}\left(\sigma_{B}\right) \oplus_{p} C_{p}^{K} \oplus_{p} R_{p}^{L}$. This finishes the proof since clearly $X_{p}\left(\sigma_{B}\right)$ is cb-isomorphic to $X_{p}(\sigma)$. Obviously the $C_{p^{-}}$and $R_{p^{-}}$-terms do not appear in case $\sigma_{n} \rightarrow 0$ and $\varrho_{n} \rightarrow 0$.

\section{Operator space properties of the matricial Rosenthal spaces.} In this section we will discuss the operator space structure of the matricial Rosenthal spaces. As before we let $p>2,1 / 2=1 / p+1 / r$, and let $\sigma$ be a sequence with $\sigma_{n} \geq 0$. $\left(\xi_{n}\right)$ denotes the unit vector basis of $\ell_{2}$. Throughout the rest of the paper we let $\mathcal{R}$ denote the hyperfinite $\mathrm{II}_{1}$ factor defined as the $\sigma$-weak closure of the infinite tensor product $\bigotimes_{n \in \mathbb{N}} M_{2}$ in the GNSconstruction with respect to the trace $\tau_{\mathcal{R}}=\bigotimes_{n \in \mathbb{N}} \operatorname{tr} / 2$.

We start with the following result on $Y_{p}(\sigma)$ :

Proposition 3.1. $Y_{p}(\sigma)$ is complemented in $L_{p}(\mathcal{R})$.

Proof. Let $\mu$ denote the Lebesgue measure on $(0, \infty)$ and let $A_{n} \subset$ $(0, \infty)$ be disjoint sets with $\mu\left(A_{n}\right)=\sigma_{n}^{r}$ for all $n \in \mathbb{N}$. We consider the subspace $V \subset L_{p}\left((0, \infty) ; S_{p}\right) \cap L_{2}^{r_{p} \cap c_{p}}\left((0, \infty) ; S_{2}\right)$ defined as the closure of $\left\{\sum_{n} \mu\left(A_{n}\right)^{-1 / p} 1_{A_{n}} x_{n} \mid x_{n} \in S_{p}^{n}\right\}$.

Given $X_{n} \in S_{p} \otimes S_{p}^{n}$, we have

$$
\left\|\sum_{n} \mu\left(A_{n}\right)^{-1 / p} 1_{A_{n}} X_{n}\right\|_{L_{p}\left(S_{p}\right)}=\left(\sum_{n}\left\|X_{n}\right\|_{p}^{p}\right)^{1 / p} .
$$

Further,

$$
\begin{aligned}
\left\|\sum_{n} \mu\left(A_{n}\right)^{-1 / p} 1_{A_{n}} X_{n}\right\|_{S_{p}\left[L_{2}^{c_{p}}\right]} & =\left\|\left(\sum_{n} \mu\left(A_{n}\right)^{1-2 / p}(\mathrm{id} \otimes \operatorname{tr})\left(X_{n}^{*} X_{n}\right)\right)^{1 / 2}\right\|_{S_{p}} \\
& =\left\|\left(\sum_{n} \sigma_{n}^{2}(\mathrm{id} \otimes \operatorname{tr})\left(X_{n}^{*} X_{n}\right)\right)^{1 / 2}\right\|_{S_{p}} \cdot
\end{aligned}
$$

The calculation for the row term is similar. Comparing this with (1.22) we conclude that $V$ is cb-isomorphic to $Y_{p}(\sigma)$.

For every $n \in \mathbb{N}$ we let $p_{n}$ denote the orthogonal projection of $\ell_{2}$ onto $\operatorname{span}\left\{\xi_{n} \mid n(n-1) / 2+1 \leq k \leq n(n+1) / 2\right\}$. Since $B=\left\{\sum_{n} 1_{A_{n}} \otimes x_{n} \mid\right.$ $\left.x_{n}=p_{n} x_{n} p_{n}\right\}$ is a von Neumann subalgebra of $L_{\infty}\left((0, \infty) ; B\left(\ell_{2}\right)\right)$ and the restriction of the trace is normal on $B$, we deduce from [28] that there is the 
conditional expectation

$$
E(x)=\sum_{n} 1_{A_{n}} \otimes \int_{A_{n}} p_{n} x(t) p_{n} \frac{d t}{\mu\left(A_{n}\right)}
$$

which is completely contractive on $L_{p}\left((0, \infty) ; S_{p}\right)$ for all $1 \leq p \leq \infty$. Clearly $E$ is a projection onto $V$ and hence $V$ is cb-complemented in $L_{p}\left((0, \infty) ; S_{p}\right) \cap$ $L_{2}^{c_{p} \cap r_{p}}\left((0, \infty) ; S_{2}\right)$. According to [9] the latter space is cb-isomorphic to $L_{p}(\mathcal{R})$, and the assertion is proved.

REMARK 3.2. According to [9], the spaces

$$
L_{p}\left((0, \infty) ; S_{p}\right) \cap L_{2}^{c_{p}}\left((0, \infty) ; S_{2}\right) \quad \text { and } \quad L_{p}\left((0, \infty) ; S_{p}\right) \cap L_{2}^{r_{p}}\left((0, \infty) ; S_{2}\right)
$$

are cb-isomorphic to completely complemented subspaces in $L_{p}\left(\mathcal{R} \otimes B\left(\ell_{2}\right)\right)$ and hence the same argument as above shows that $Y_{p, c_{p}}$ and $Y_{p, r_{p}}$ are cbisomorphic to cb-complemented subspaces of $L_{p}\left(\mathcal{R} \otimes B\left(\ell_{2}\right)\right)$. However, in general we cannot expect a cb-embedding into $L_{p}(\mathcal{R})$. Indeed, from Theorem 1.5 it follows that if $\sigma$ satisfies (1.5) and (1.6), then $S_{p}$ cb-embeds into $Z_{p}(\sigma)$ but it does not embed into $L_{p}(\mathcal{R})$ according to a result of Sukochev [27]. Hence $Z_{p}(\sigma)$ does not cb-embed into $L_{p}(\mathcal{R})$.

Corollary 3.3. The spaces $Y_{p}(\sigma), Y_{p, c_{p}}(\sigma)$, and $Y_{p, r_{p}}(\sigma)$ have the $\gamma_{p}-A P$.

Proof. Since $L_{p}\left(\mathcal{R} \otimes B\left(\ell_{2}\right)\right)$ is the $L_{p}$-space of an injective von Neumann algebra, this space has the $\gamma_{p}$ AP. The $\gamma_{p}$ AP passes to complemented subspaces.

We now turn our attention to the space $Z_{p}(\sigma)$ but for this we need some preliminary results.

Let $m, n \in \mathbb{N}$ and let $D$ be a positive $m \times m$ diagonal matrix with $\operatorname{tr}(D)=1$. We define $Z_{p}^{m}(n, D)$ to be the subspace of $S_{p}^{m} \oplus_{p} C_{p}^{m^{2}} \oplus_{p} R_{p}^{m^{2}}$ defined by

$$
Z_{p}^{m}(n, D)=\left\{\left(x, n^{1 / r} x D^{1 / r}, n^{1 / r} D^{1 / r} x\right) \mid x \in S_{P}^{m}\right\}
$$

Here we consider $x D^{1 / r}$ as an element of $C_{p}^{m}\left(C_{p}^{m}\right)=C_{p}^{m^{2}}$, and $D^{1 / r} x$ as an element of $R_{p}^{m}\left(R_{p}^{m}\right)=R_{p}^{m^{2}}$. The spaces $Z_{p, c_{p}}^{m}(n, D)$ and $Z_{p, r_{p}}^{m}(n, D)$ are defined similarly as subspaces of $S_{p}^{m} \oplus_{p} C_{p}^{m^{2}}$, respectively $S_{p}^{m} \oplus_{p} R_{p}^{m^{2}}$.

For every $1 \leq i \leq n$ we define $\Psi_{i}: S_{p}^{m} \rightarrow S_{p}^{m^{n}}=S_{p}^{\otimes_{n}}$ by

$$
\Psi_{i}(x)=D^{1 / p} \otimes \cdots \otimes D^{1 / p} \otimes x \otimes D^{1 / p} \otimes \cdots \otimes D^{1 / p}
$$

for all $x \in S_{p}^{m}$, where $x$ is the $i$ th factor. Further, we put

$$
U_{p}(x)=n^{-1 / p} \sum_{i=1}^{n} \varepsilon_{i} \Psi_{i}(x) \quad \text { for all } x \in S_{p}^{m},
$$




$$
\begin{array}{ll}
U_{p, c}(x)=n^{-1 / p} \sum_{i=1}^{n} \varepsilon_{i} \Psi_{i}(x) \otimes e_{i 1} & \text { for all } x \in S_{p}^{m}, \\
U_{p, r}(x)=n^{-1 / p} \sum_{i=1}^{n} \varepsilon_{i} \Psi_{i}(x) \otimes e_{1 i} & \text { for all } x \in S_{p}^{m},
\end{array}
$$

where $\left(\varepsilon_{i}\right)$ is the sequence of Rademacher functions on $[0,1]$.

THEOREM 3.4. $U_{p}$ acts as a cb-isomorphism of $Z_{p}^{m}(n, D)$ onto its image which is cb-complemented in $L_{p}\left([0,1] ; S_{p}^{m^{n}}\right)$ with cb-norms only depending on $p$. Similarly $Z_{p, c_{p}}^{m}(n, D)$ (resp., $\left.Z_{p, r_{p}}^{m}(n, D)\right)$ is cb-complemented in $L_{p}\left([0,1] ; S_{p}^{m^{n}} \otimes C_{p}^{n}\right)$ (resp., $\left.L_{p}\left([0,1] ; S_{p}^{m^{n}} \otimes R_{p}^{n}\right)\right)$ via the map $U_{p, c}$ (resp., $\left.U_{p, r}\right)$.

Proof. Let $\left\{x_{j k} \mid 1 \leq j, k \leq m\right\} \subseteq S_{p}$. For every $1 \leq i \leq n$ put

$$
Y_{i}=\varepsilon_{i} \sum_{j, k} x_{j} k \otimes \Psi_{i}\left(e_{j k}\right) .
$$

Then the $Y_{i}$ 's are independent in the sense of [14] and have mean zero.

Therefore, if we put $E(x \otimes y)=\operatorname{tr}\left(D^{1-2 / p} x\right) y$ for all $x \in S_{p}^{m^{n}}$ and all $y \in S_{p}$ and let " " denote a two-sided inequality with constants only depending on $p$, then [14, Theorem 1.2] gives

$$
\begin{aligned}
\left\|\sum_{j, k} x_{j k} \otimes U_{p}\left(e_{j k}\right)\right\|_{S_{p}\left[L_{p}\left(S_{p}^{m^{n}}\right)\right]}=n^{-1 / p} \| & \sum_{i=1}^{n} Y_{i} \|_{S_{p}\left[L_{p}\left(S_{p}^{m^{n}}\right)\right]} \\
\sim n^{-1 / p} \max \left\{\left(\sum_{i=1}^{n}\left\|Y_{i}\right\|_{S_{p}\left[L_{p}\left(S_{p}^{m^{n}}\right)\right]}^{p}\right)^{1 / p},\right. & \left\|\left(\sum_{i=1}^{n} E\left(Y_{i}^{*} Y_{i}\right)\right)^{1 / p}\right\|_{S_{p}}, \\
& \left.\left\|\left(\sum_{i=1}^{n} E\left(Y_{i} Y_{i}^{*}\right)\right)^{1 / p}\right\|_{S_{p}}\right\} .
\end{aligned}
$$

For all $i \leq n$ we easily get

$$
\left\|Y_{i}\right\|_{S_{p}\left[L_{p}\left(S_{p}^{m^{n}}\right)\right]}=\left\|\sum_{j, k} x_{j k} \otimes e_{j k}\right\|_{S_{p}\left[S_{p}^{m}\right]} .
$$

Further,

$$
\begin{aligned}
\left\|\left(\sum_{i=1}^{n} E\left(Y_{i}^{*} Y_{i}\right)\right)^{1 / 2}\right\|_{S_{p}} & =n^{1 / 2}\left\|\left(\sum_{j, k} \sigma_{k}^{1-2 / p} x_{j k}^{*} x_{j k}\right)^{1 / 2}\right\|_{S_{p}} \\
& =n^{1 / 2}\left\|\sum_{k=1}^{m} \sigma_{k}^{1 / r} \sum_{j=1}^{m} x_{j k} \otimes e_{j k}\right\|_{S_{p}\left[C_{p}^{m^{2}}\right]}
\end{aligned}
$$


and similarly

$$
\left\|\left(\sum_{i=1}^{n} E\left(Y_{i} Y_{i}^{*}\right)\right)^{1 / 2}\right\|_{S_{p}}=n^{1 / 2}\left\|\sum_{j=1}^{n} \sigma_{j}^{1 / r} \sum_{k=1}^{m} x_{j k} \otimes e_{j k}\right\|_{S_{p}\left[R_{p}^{m^{2}}\right]} .
$$

Combining these calculations with (3.1) we deduce that $U$ is a cb-isomorphism of $Z_{p}^{m}(n, D)$ onto its image.

For every $1 \leq i \leq n$ we define $\Psi_{i}^{\prime}: S_{p^{\prime}}^{m} \rightarrow S_{p^{\prime}}^{m^{n}}$ by

$$
\Psi_{i}^{\prime}(x)=D^{1 / p} \otimes \cdots \otimes D^{1 / p} \otimes x \otimes D^{1 / p} \otimes \cdots \otimes D^{1 / p}
$$

for every $x \in S_{p^{\prime}}$, where $x$ is the $i$ th factor, and $U_{p^{\prime}}=\sum_{i=1}^{n} \varepsilon_{i} \Psi_{i}^{\prime}(x)$ for all $x \in S_{p^{\prime}}$. Using [14, Theorem 4.3] we can show in a similar manner that $U_{p^{\prime}}$ acts as a cb-bounded operator from $Z_{p}^{m}(n, D)^{*}$ to $L_{p^{\prime}}\left([0,1] ; S_{p}^{m^{n}}\right)$ It is readily verified that $U_{p} U_{p^{\prime}}^{*}$ is a cb-bounded projection of $L_{p}\left([0,1] ; S_{p}^{m^{n}}\right)$ onto the range of $U_{p}$.

The argument for $U_{p, c}$ and $U_{p, r}$ is similar.

We are now able to prove:

THEOREM 3.5. Let $2 \leq p, r<\infty$ be such that $1 / 2=1 / p+1 / r$. If $\sigma$ is a sequence of positive numbers such that $\sigma \notin \ell_{r}$ and $\liminf _{n} \sigma_{n}=0$, then $Y_{p}(\sigma), Y_{p, r_{p}}(\sigma), Y_{p, c_{p}}(\sigma), Z_{p}(\sigma), Z_{p, r}(\sigma)$, and $Z_{p, c}(\sigma)$ are $\mathcal{C O} \mathcal{S}_{p}$-spaces.

Proof. Consider

$$
s_{j}=\sum_{k=1}^{j} \sigma_{k}^{r}
$$

By assumption $s_{j}$ tends to $\infty$ and hence we can find a subsequence $\left(j_{k}\right)$ and integers $n_{k}$ such that

$$
n_{k} \leq s_{j_{k}} \leq n_{k}+1
$$

By definition $Z_{p}, Z_{p, c}, Z_{p, r}$ are the closures of $\bigcup_{k} Z_{p}^{j_{k}}, \bigcup_{k} Z_{p, c}^{j_{k}}, \bigcup_{k} Z_{p, r}^{j_{k}}$, respectively. Fix $k \in \mathbb{N}$ and define $\varrho_{k}=s_{j_{k}}^{-1}\left(\sigma_{j}^{r}\right)_{j \leq j_{k}}$. The map

$$
w(x)=\left(x, n_{k}^{1 / r} x D_{\varrho_{k}}^{1 / r}, n_{k}^{1 / r} D_{\varrho_{k}}^{1 / r} x\right)
$$

yields an isomorphism between $Z_{p}^{j_{k}}(\sigma)$ and $Z_{p}\left(n_{k}, D_{\varrho_{k}}\right)$. Indeed, for $\sigma_{k}=$ $\left(\sigma_{j}\right)_{j \leq j_{k}}$ we have

$$
n_{k}^{1 / r} D_{\varrho_{k}}^{1 / r}=\left(\frac{n_{k}}{s_{j_{k}}}\right)^{1 / r} D_{\sigma_{k}}
$$

and

$$
1 \leq\left(\frac{n_{k}}{s_{j_{k}}}\right)^{1 / r} \leq\left(1+\frac{1}{n_{k}}\right)^{1 / r} \leq 2 .
$$

Hence by Theorem 3.4, $Z_{p}^{j_{k}}(\sigma)$ has the $\gamma_{p}$-AP with a constant only depending $\sigma$ and $p$, and therefore $Z_{p}(\sigma)$ has the $\gamma_{p}$-AP. Similarly for $Z_{p, c_{p}}(\sigma)$ and 
$Z_{p, r_{p}}(\sigma)$. The spaces $Y_{p}(\sigma), Y_{p, c_{p}}(\sigma)$, and $Y_{p, r_{p}}(\sigma)$ have the $\gamma_{p}$-AP by Corollary 3.3. Since $\liminf \sigma_{n}=0$, we can find a subsequence $\sigma^{\prime}=\sigma_{n_{k}}$ such that $\left(\sigma_{n_{k}}\right) \in \ell_{r}$. Then the map $M_{r}: S_{p} \rightarrow C_{p}\left(\mathbb{N}^{2}\right)$ defined by $M_{r}(x)=$ $x D_{\sigma^{\prime}}$ is completely bounded, and similarly $M_{l}: S_{p} \rightarrow R_{p}\left(\mathbb{N}^{2}\right)$ defined by $L_{l}(x)=D_{\sigma^{\prime}} x$ is completely bounded. If $A=\left\{n_{k}: k \in \mathbb{N}\right\}$, then the subspace $Z_{A}=\left\{\left(x_{i j}\right) \mid i, j \in A\right\}$ is cb-isomorphic to $S_{p}$ and complemented in $Z_{p}(\sigma), Z_{p, c}(\sigma)$, and $Z_{p, r}(\sigma)$, respectively. By the definition of $Y_{p}(\sigma)$ we deduce that $Y_{A}=\left\{\left(x_{k}\right)_{k} \mid k \in A, x_{k} \in M_{m_{k}}\right\}$ is cb-isomorphic to $\left(\sum_{k \in A} \oplus_{p} S_{p}^{m_{k}}\right)_{p}$ and cb-complemented. Thus all these spaces contain $S_{p}^{n}$ 's uniformly complemented. According to [11, Theorem 2.2], these spaces are $\mathcal{C O S}_{p}$-spaces.

4. Uncomplemented copies of some $\mathcal{O} \mathcal{L}_{p}$-spaces. Throughout this section, $2<p<\infty$, unless specified otherwise.

THEOREM 4.1. Let $X$ and $Y$ be subspaces of rectangular $\mathcal{O} \mathcal{L}_{p}$-spaces such that $X$ is completely isomorphic to a subspace of $Y$. Then $\ell_{p}(Y)$ (respectively, $\left.S_{p}[Y]\right)$ contains an uncomplemented completely isomorphic copy of $\ell_{p}(X)$ (respectively, $\left.S_{p}[X]\right)$.

Before proving the theorem, we formulate a corollary of it.

Corollary 4.2 .

(a) Suppose $X$ is one of the following operator spaces: $\ell_{p}, S_{p}, \mathcal{K}_{p}$, or $L_{p}(\mathcal{R})$. Then $X$ contains an uncomplemented copy of itself.

(b) Suppose $\mathcal{N}$ is a group von Neumann algebra with $Q W E P$, and $X$ is either $\ell_{p}\left(L_{p}(\mathcal{N})\right)$ or $S_{p}\left[L_{p}(\mathcal{N})\right]$. Then $X$ contains an uncomplemented copy of itself.

Proof. All the spaces listed in (a) and (b) are $\mathcal{O} \mathcal{L}_{p}$-spaces (see [12] for the spaces from (b)). Moreover, any of the spaces $X$ listed in (a) is completely isomorphic to $\ell_{p}(X)$, by Pełczyński's decomposition method. The same argument shows that for $\mathcal{N}$ as in (b), $S_{p}\left[L_{p}(\mathcal{N})\right]$ is completely isomorphic to $\ell_{p}\left(S_{p}\left[L_{p}(\mathcal{N})\right]\right)$.

To establish Theorem 4.1, consider a finite-dimensional version of the Rosenthal space. More precisely, if $\sigma=\left(\sigma_{n}\right)_{n \in \mathbb{N}}$ is a sequence of positive numbers, then we let $X_{p}^{m}(\sigma)$ be the linear span of the first $m$ vectors of the canonical basis of $X_{p}(\sigma)$. By Corollary 2.2 there exist $\lambda>0$ and a sequence $\left(k_{m}\right)_{m \in \mathbb{N}}$ such that $\ell_{p}^{k_{m}}$ contains a $\lambda$-completely complemented $\lambda$-completely isomorphic copy of $X_{p}^{m}(\sigma)$.

Now suppose the sequence $\left(\sigma_{n}\right)$ satisfies (1.5) and (1.6). By [26], if $P_{m}$ is a projection from $\ell_{p}^{m} \oplus_{p} R_{p}^{m} \oplus_{p} C_{p}^{m}$ onto the "natural" copy of $X_{p}^{m}(\sigma)$, then $\lim _{m}\left\|P_{m}\right\|=\infty$. By [20] (see also [23]), $\ell_{p}^{m} \oplus_{p} R_{p}^{m} \oplus_{p} C_{p}^{m}$ embeds into $\ell_{p}^{3^{m}} \quad c_{p}$-completely isomorphically. Thus, there exists a sequence $\left(T_{m}\right)$ 
of complete contractions $T_{m}: X_{p}^{m}(\sigma) \rightarrow \ell_{p}^{3^{m}}$ such that $\left\|T_{m}^{-1}\right\|_{\mathrm{cb}} \leq c_{p}$, and $\lim _{m}\left\|Q_{m}\right\|=\infty$ whenever $Q_{m}$ is a projection from $\ell_{p}^{3^{m}}$ onto range $\left(T_{m}\right)$.

The properties of the spaces $X_{p}^{m}(\sigma)$ yield:

LEMMA 4.3. $\ell_{p}$ contains an uncomplemented completely isomorphic copy of itself.

Proof. Suppose the sequence $\left(\sigma_{m}\right)$ satisfies (1.5) and (1.6). Consider the spaces $Y=\left(\sum_{m} \ell_{p}^{3^{m}}\right)_{p}$ and $Z=\left(\sum_{m} T_{m}\left(X_{p}^{m}(\sigma)\right)\right)_{p}$. By the discussion preceding the statement of this lemma, $Z$ is an uncomplemented subspace of $Y$. Moreover, $Y$ is completely isometric to $\ell_{p}$. It remains to show that $Z$ is completely isomorphic to $\ell_{p}$. To this end, note that $Z$ is completely isomorphic to a completely complemented subspace of $\left(\sum_{m} \ell_{p}^{k_{m}}\right)_{p} \sim \ell_{p}$. Moreover, $Y$ contains a completely complemented copy of $\ell_{p}$. As $\ell_{p}=\ell_{p}\left(\ell_{p}\right)$, we complete the proof by applying the Pełczyński decomposition method.

We need yet another lemma.

Lemma 4.4. Suppose $X$ is a rectangular $\mathcal{O} \mathcal{L}_{p}$-space, and $T$ is a complete isomorphism from $\ell_{p}$ onto a subspace. Then $T \otimes \mathrm{id}_{X}$ is a complete isomorphism from $\ell_{p}(X)$ onto its range, viewed as a subspace of $\ell_{p}(X)$.

Proof. We can assume that $T$ is a complete contraction and let $c=$ $\left\|T^{-1}\right\|_{\mathrm{cb}}$. It suffices to show that $T \otimes \mathrm{id}_{S_{p}^{N}}: \ell_{p}\left(S_{p}^{N}\right) \rightarrow \ell_{p}\left(S_{p}^{N}\right)$ is a complete contraction, and $\left\|\left(T \otimes \mathrm{id}_{S_{p}^{N}}\right)^{-1}\right\|_{c b} \leq c$. To complete the proof identify $\ell_{p}\left(S_{p}^{N}\right)$ with $S_{p}^{N}\left[\ell_{p}\right]$ and apply Proposition 0.1 .

REMARK 4.5. The same result also holds for complete isomorphisms from $S_{p}$ onto its subspaces.

Proof of Theorem 4.1 Suppose $X$ and $Y$ are subspaces of rectangular $\mathcal{O} \mathcal{L}_{p^{-}}$-spaces and $S: X \rightarrow Y$ is a complete isomorphism. Let $T: \ell_{p} \rightarrow \ell_{p}$ be a complete isomorphism with an uncomplemented range (such a $T$ exists, by Lemma 4.3). By Lemma 4.4, $T \otimes S$ determines a complete isomorphism from $\ell_{p}(X)$ onto a subspace of $\ell_{p}(Y)$. It remains to show that range $(T \otimes S)$ is uncomplemented. Indeed, suppose for the sake of contradiction that there exists a projection $P$ from $\ell_{p}(Y)$ onto range $(T \otimes S)$. Pick $x \in X \backslash\{0\}$ and denote by $Q$ a bounded projection onto $\operatorname{span}(S x)$. As $T$ is a complete isomorphism, $\widetilde{Q}=I_{\operatorname{range}(T)} \otimes Q$ is a completely bounded projection from range $(T \otimes S)$ onto range $(T) \otimes \operatorname{span}(S x)$. Hence $\left.\widetilde{Q} \circ P\right|_{\ell_{p} \otimes \operatorname{span}(S x)}$ is a bounded projection from $\ell_{p} \otimes \operatorname{span}(S x)$ onto range $(T) \otimes \operatorname{span}(S x)$, which contradicts the fact that range $(T)$ is uncomplemented.

Corollary 4.6. Suppose $\mathcal{N}$ is a von Neumann algebra equipped with a normal semifinite faithful trace which is not of type $I$. Then there exists an uncomplemented subspace $X$ of $L_{p}(\mathcal{N})$ completely isomorphic to $L_{p}(\mathcal{R})$. 
Proof. By [11] (see also [21]), $L_{p}(\mathcal{N})$ contains a (completely contractively complemented) subspace $Y$, completely isometric to $L_{p}(\mathcal{R})$. By Theorem 4.1, $Y$ contains an uncomplemented copy of $L_{p}(\mathcal{R})$.

Corollary 4.7.

(1) Every infinite-dimensional rectangular $\mathcal{O} \mathcal{L}_{p}$-space contains an uncomplemented copy of $\ell_{p}$.

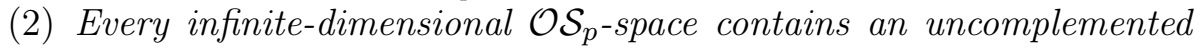
copy of $\left(\sum_{n} S_{p}^{n}\right)_{p}$.

Proof. By [11] any $\mathcal{O} \mathcal{L}_{p}$-space $X$ (with $1<p<\infty$ ) embeds completely isometrically (and even completely contractively complementedly) into $\prod_{\mathcal{U}} S_{p}$, where $\mathcal{U}$ is an ultrafilter. By [24] and [25], $X$ contains a completely isomorphic (and even completely complemented) subspace $Y$, completely isomorphic to $\ell_{p}$. Moreover, if $X$ is an $\mathcal{O} \mathcal{S}_{p}$-space, then it contains a subspace $Y$, completely isomorphic to $\left(\sum_{n} S_{p}^{n}\right)_{p}$. In either case an application of Theorem 4.1 completes the proof.

\section{References}

[1] J. Arazy, On large subspaces of the Schatten p-classes, Compos. Math. 41 (1980), 297-336.

[2] J. Arazy and J. Lindenstrauss, Some linear topological properties of the spaces $C_{p}$ of operators on Hilbert space, ibid. 30 (1975), 81-111.

[3] J. Bourgain, H. P. Rosenthal, and G. Schechtman, An ordinal $L_{p}$-index for Banach spaces, with application to complemented subspaces of $L_{p}$, Ann. of Math. 114 (1981), 193-228.

[4] E. G. Effros and Z.-J. Ruan, $\mathcal{O L}_{p}$ spaces, in: Contemp. Math. 228, Amer. Math. Soc., 1998, 51-77.

[5] - - -, Operator Spaces, London Math. Soc. Monogr. 23, Oxford Univ. Press, 2000.

[6] U. Haagerup, H. P. Rosenthal and F. A. Sukochev, Banach embedding properties of non-commutative $L_{p}$-spaces, Mem. Amer. Math. Soc. 163 (2003), no. 766.

[7] F. Hansen and G. K. Pedersen, Perturbation formulas for traces on $C^{*}$-algebras, Publ. RIMS Kyoto Univ. 31 (1995), 169-178.

[8] W. B. Johnson, B. Maurey, G. Schechtman, and L. Tzafriri, Symmetric structures in Banach spaces, ibid. 217 (1979).

[9] M. Junge, Doob's inequality for non-commutative martingales, J. Reine Angew. Math. 549 (2002), 149-190.

[10] - Fubini's theorem for ultraproducts of non-commutative $L_{p}$-spaces, Canad. J. Math. 56 (2004), 983-1021.

[11] M. Junge, N. J. Nielsen, Z.-J. Ruan, and Q. Xu, $\mathcal{O} \mathcal{L}_{p}$ spaces-The local structure of non-commutative $L_{p}$-spaces I, Adv. Math. 187 (2004), 257-319.

[12] M. Junge and Z.-J. Ruan, Approximation properties for non-commutative $L_{p}$-spaces associated with discrete groups, Duke Math. J. 117 (2003), 313-341.

[13] M. Junge and Q. Xu, Non-commutative Burkholder-Rosenthal inequalities, Ann. Probab. 31 (2003), 948-995. 
[14] M. Junge and Q. Xu, Non-commutative Burkholder-Rosenthal inequalities II: Applications, preprint.

[15] R. Kadison and J. Ringrose, Fundamentals of the Theory of Operator Algebras, Vol. II. Advanced Theory, Amer. Math. Soc., Providence, RI, 1997.

[16] J.-P. Kahane, Some Random Series of Functions, 2nd ed., Cambridge Univ. Press, Cambridge, 1985.

[17] J. Lindenstrauss and H. P. Rosenthal, The $\mathcal{L}_{p}$-spaces, Israel J. Math. 7 (1969), 325-349.

[18] J. Lindenstrauss and L. Tzafriri, Classical Banach Spaces I. Sequence Spaces, Ergeb. Math. Grenzgeb. 92, Springer, Berlin, 1977.

[19] - - -, Classical Banach Spaces II. Function Spaces, Ergeb. Math. Grenzgeb. 97, Springer, Berlin, 1979.

[20] F. Lust-Piquard, Inégalités de Khintchine dans $C_{p}(1<p<\infty)$, C. R. Acad. Sci. Paris 303 (1986), 289-292.

[21] J. Marcolino, La stabilité des espaces $L_{p}$ non-commutatifs, Math. Scand. 81 (1997), 212-219.

[22] G. Pisier, Some results on Banach spaces without local unconditional structure, Compos. Math. 37 (1978), 3-19.

[23] - Non-Commutative Vector Valued $L_{p}$-Spaces and Completely $p$-Summing Maps, Astérisque 247 (1998).

[24] Y. Raynaud, On ultrapowers of non-commutative $L_{p}$ spaces, J. Operator Theory 48 (2002), 41-68.

[25] Y. Raynaud and Q. Xu, On subspaces of non-commutative $L_{p}$-spaces, J. Funct. Anal. 203 (2003), 149-196.

[26] H. P. Rosenthal, On the subspaces of $L_{p}(p>2)$ spanned by sequences of independent random variables, Israel J. Math. 8 (1970), 273-303.

[27] F. A. Sukochev, Non-isomorphisms of $L_{p}$-spaces associated with finite or infinite von Neumann algebras, Proc. Amer. Math. Soc. 124 (1996), 1517-1527.

[28] M. Takesaki, Theory of Operator Algebras I, Springer, New York, 2001.

Department of Mathematics

University of Illinois at Urbana-Champaign

1409 W. Green Street

Urbana, IL 61801, U.S.A.

E-mail: junge@math.uiuc.edu

Department of Mathematics

University of California, Irvine

103 MSTB

Irvine, CA 92697-3875, U.S.A.

E-mail: toikhber@math.uci.edu
Department of Mathematics and Computer Science University of Southern Denmark Campusvej 55

DK-5230 Odense M, Denmark E-mail: njn@imada.sdu.dk

Received January 17, 2007

Revised version February 27, 2008 